\title{
On quartic half-arc-transitive metacirculants
}

\author{
Dragan Marušič • Primož Šparl
}

Received: 7 June 2007 / Accepted: 6 November 2007 / Published online: 5 December 2007

(C) Springer Science+Business Media, LLC 2007

\begin{abstract}
Following Alspach and Parsons, a metacirculant graph is a graph admitting a transitive group generated by two automorphisms $\rho$ and $\sigma$, where $\rho$ is $(m, n)$ semiregular for some integers $m \geq 1, n \geq 2$, and where $\sigma$ normalizes $\rho$, cyclically permuting the orbits of $\rho$ in such a way that $\sigma^{m}$ has at least one fixed vertex. A halfarc-transitive graph is a vertex- and edge- but not arc-transitive graph. In this article quartic half-arc-transitive metacirculants are explored and their connection to the so called tightly attached quartic half-arc-transitive graphs is explored. It is shown that there are three essentially different possibilities for a quartic half-arc-transitive metacirculant which is not tightly attached to exist. These graphs are extensively studied and some infinite families of such graphs are constructed.
\end{abstract}

Keywords Graph · Metacirculant graph · Half-arc-transitive · Tightly attached · Automorphism group

\section{Introductory and historic remarks}

Throughout this paper graphs are assumed to be finite and, unless stated otherwise, simple, connected and undirected (but with an implicit orientation of the edges when appropriate). For group-theoretic concepts not defined here we refer the reader to $[4,9,34]$, and for graph-theoretic terms not defined here we refer the reader to [5].

Both authors were supported in part by "ARRS - Agencija za znanost Republike Slovenije”, program no. P1-0285.

D. Marušič $(\bowtie)$

University of Primorska, FAMNIT, Glagoljaška 8, 6000 Koper, Slovenia

e-mail: dragan.marusic@guest.arnes.si

D. Marušič · P. Šparl

IMFM, University of Ljubljana, Jadranska 19, 1111 Ljubljana, Slovenia 
Given a graph $X$ we let $V(X), E(X), A(X)$ and Aut $X$ be the vertex set, the edge set, the arc set and the automorphism group of $X$, respectively. A graph $X$ is said to be vertex-transitive, edge-transitive and arc-transitive if its automorphism group Aut $X$ acts transitively on $V(X), E(X)$ and $A(X)$, respectively. We say that $X$ is halfarc-transitive provided it is vertex- and edge- but not arc-transitive. More generally, by a half-arc-transitive action of a subgroup $G \leq$ Aut $X$ on $X$ we mean a vertex-and edge- but not arc-transitive action of $G$ on $X$. In this case we say that the graph $X$ is $\left(G, \frac{1}{2}\right)$-arc-transitive, and we say that the graph $X$ is $\left(G, \frac{1}{2}, H\right)$-arc-transitive when it needs to be stressed that the vertex stabilizers $G_{v}$ (for $\left.v \in V(X)\right)$ are isomorphic to a particular subgroup $H \leq G$. By a classical result of Tutte [32, 7.35, p. 59], a graph admitting a half-arc-transitive group action is necessarily of even valency. A few years later Tutte's question as to the existence of half-arc-transitive graphs of a given even valency was answered by Bouwer [6] with a construction of a $2 k$-valent halfarc-transitive graph for every $k \geq 2$. The smallest graph in Bouwer's family has 54 vertices and valency 4. Doyle [10] and Holt [15] independently found one with 27 vertices, a graph that is now known to be the smallest half-arc-transitive graph [3].

Interest in the study of this class of graphs reemerged about a decade later following a series of papers dealing mainly with classification of certain restricted classes of such graphs as well as with various methods of constructions of new families of such graphs [2, 3, 30, 31, 33, 36]; but see also the survey article [21] which covers the respective literature prior to 1998 . With some of the research emphasis shifting to questions concerning structural properties of half-arc-transitive graphs, these graphs have remained an active topic of research to this day; see [7, 8, 11-14, 16-18, 20, 22-29, 35, 37].

In view of the fact that 4 is the smallest admissible valency for a half-arc-transitive graph, special attention has rightly been given to the study of quartic half-arctransitive graphs. One of the possible approaches in the investigation of their properties concerns the so called "attachment of alternating cycles" question. Layed out in [20], the underlying theory is made up of the following main ingredients. For a quartic graph $X$ admitting a half-arc-transitive action of some subgroup $G$ of Aut $X$, let $D_{G}(X)$ be one of the two oriented graphs associated in a natural way with the action of $G$ on $X$. (In other words, $D_{G}(X)$ is an orbital graph of $G$ relative to a nonself-paired orbital associated with a non-self-paired suborbit of length 2 and $X$ is its underlying undirected graph.) An even length cycle $C$ in $X$ is a $G$-alternating $c y$ cle if every other vertex of $C$ is the tail and every other vertex of $C$ is the head (in $\left.D_{G}(X)\right)$ of its two incident edges. It was shown in [20] that, first, all $G$-alternating cycles of $X$ have the same length - half of this length is called the G-radius of $X$ - and second, that any two adjacent $G$-alternating cycles intersect in the same number of vertices, called the $G$-attachment number of $X$. The intersection of two adjacent $G$-alternating cycles is called a $G$-attachment set. The attachment of alternating cycles concept has been addressed in a number of papers [20, 27-29, 35] with a particular attention given to the so called $G$-tightly attached graphs, that is, graphs where two adjacent $G$-alternating cycles have every other vertex in common. In other words, their $G$-attachment number coincides with $G$-radius. In all the above definitions the symbol $G$ is omitted when $G=$ Aut $X$. Tightly attached graphs with odd radius have been completely classified in [20], whereas the classification of tightly attached graphs with even radius, dealt with also in $[13,27,35]$, has been very recently 
completed in [29]. At the other extreme, graphs with $G$-attachment number equal to 1 and 2, respectively, are called $G$-loosely attached graphs and $G$-antipodally attached graphs. As shown in [28], there exist infinite families of quartic half-arc-transitive graphs with arbitrarily prescribed attachment numbers. However, in view of the fact that every quartic half-arc-transitive graph may be obtained as a cover of a loosely, antipodally or tightly attached graph [27], it is these three families of graphs that deserve special attention.

Now, as it turns out, all tightly attached quartic half-arc-transitive graphs are metacirculant graphs [20]. (For the definition of a metacirculant graph see Section 2.) The connection between the two classes of graphs goes so far as to suggest that even if quartic half-arc-transitive metacirculants which are not tightly attached do exist, constructing them will not be an easy task. Exploring this connection is the main aim of this article. Although short of a complete classification of quartic half-arc-transitive metacirculants, we obtain a description of the three essentially different possibilities for a quartic half-arc-transitive metacirculant which is not tightly attached to exist, together with constructions of infinite families of such graphs. In doing so we give a natural decomposition of quartic half-arc-transitive metacirculants into four classes depending on the structure of the quotient circulant graph relative to the semiregular automorphism $\rho$. Loosely speaking, Class I consists of those graphs whose quotient graph is a "double-edged" cycle, Class II consists of graphs whose quotient is a cycle with a loop at each vertex, Class III consists of graphs whose quotient is a circulant of even order with antipodal vertices joined by a double edge, and Class IV consists of graphs whose quotient is a quartic circulant which is a simple graph (see Figure 1).

The paper is organized as follows. Section 2 contains some terminology together with four infinite families of quartic metacirculants, playing an essential role in the rest of the paper. Section 3 gives the above mentioned decomposition. Section 4 is devoted to Class I graphs; in particular it is shown that this class coincides with the class of tightly attached graphs (see Theorem 4.1). Next, Section 5 deals with Class II graphs. A characterization of the graphs of this class which are not tightly attached is given (see Theorem 5.1) enabling us to construct an infinite family of such graphs (see Construction 5.10). Moreover, a list of all quartic half-arc-transitive metacirculants of Class II, of order at most 1000, that are not tightly attached is given. Finally, in Section 6 a construction of an infinite family of loosely attached (and thus not tightly attached) half-arc-transitive metacirculants of Class IV is given.

\section{Definitions and examples}

We start by some notational conventions used throughout this paper. Let $X$ be a graph. The fact that $u$ and $v$ are adjacent vertices of $X$ will be denoted by $u \sim v$; the corresponding edge will be denoted by $[u, v]$, in short by $u v$. In an oriented graph the fact that the edge $u v$ is oriented from $u$ to $v$ will be denoted by $u \rightarrow v$ (as well as by $v \leftarrow u$ ). In this case the vertex $u$ is referred to as the tail and $v$ is referred to as the head of the edge $u v$. Let $U$ and $W$ be disjoint subsets of $V(X)$. The subgraph of $X$ induced by $U$ will be denoted by $X[U]$; in short, by $[U]$, when the graph $X$ is clear from the context. Similarly, we let $X[U, W]$ (in short $[U, W]$ ) denote the 
bipartite subgraph of $X$ induced by the edges having one endvertex in $U$ and the other endvertex in $W$. Furthermore, if $\rho$ is an automorphism of $X$, we denote the corresponding quotient (multi)graph relative to $\rho$, whose vertex set is the set of orbits of $\rho$ with two orbits adjacent whenever there is an edge in $X$ joining vertices from these two orbits, by $X_{\rho}$.

For the sake of completeness we include the definition of a Cayley graph. Given a group $G$ and an inverse closed subset $S \subseteq G \backslash\{1\}$ the Cayley graph $\operatorname{Cay}(G, S)$ is the graph with vertex set $G$ and edges of the form $[g, g s]$, where $g \in G, s \in S$.

Let $m \geq 1$ and $n \geq 2$ be integers. An automorphism of a graph is called $(m, n)$ semiregular if it has $m$ orbits of length $n$ and no other orbit. We say that a graph $X$ is an $(m, n)$-metacirculant graph (in short an $(m, n)$-metacirculant) if there exists an $(m, n)$-semiregular automorphism $\rho$ of $X$, together with an additional automorphism $\sigma$ of $X$ normalizing $\rho$, that is,

$$
\sigma^{-1} \rho \sigma=\rho^{r} \quad \text { for some } \quad r \in \mathbb{Z}_{n}^{*},
$$

and cyclically permuting the orbits of $\rho$ in such a way that $\sigma^{m}$ fixes a vertex of $X$. (Hereafter $\mathbb{Z}_{n}$ denotes the ring of residue classes modulo $n$ as well as the additive cyclic group of order $n$, depending on the context.) Note that this implies that $\sigma^{m}$ fixes a vertex in every orbit of $\rho$. To stress the role of these two automorphisms in the definition of the metacirculant $X$ we shall say that $X$ is an $(m, n)$-metacirculant relative to the ordered pair $(\rho, \sigma)$. Obviously, a graph is an $(m, n)$-metacirculant relative to more than just one ordered pair of automorphisms except for the trivial case when $m=1$ and $n=2$, which corresponds to $X \cong K_{2}$. For example, the automorphism $\sigma$ may be replaced by $\sigma \rho$. A graph $X$ is a metacirculant if it is an $(m, n)$-metacirculant for some $m$ and $n$. This definition is equivalent with the original definition of a metacirculant by Alspach and Parsons (see [1]). For the purposes of this paper we extend this definition somewhat. We say that a graph $X$ is a weak $(m, n)$-metacirculant (more precisely a weak $(m, n)$-metacirculant relative to the ordered pair $(\rho, \sigma))$ if it has all the properties of an $(m, n)$-metacirculant except that we do not require that $\sigma^{m}$ fixes a vertex of $X$. We say that $X$ is a weak metacirculant if it is a weak $(m, n)$-metacirculant for some positive integers $m$ and $n$.

Note that there exist integers $m, n$ and weak $(m, n)$-metacirculants which are not $(m, n)$-metacirculants. For example, the graph $\mathcal{Y}(10,100 ; 11,90)$ (see Example 2.3 below) is a weak $(10,100)$-metacirculant but it can be seen that it is not a $(10,100)$ metacirculant. However, this graph is also a $(40,25)$-metacirculant. The question remains if the class of weak metacirculants is indeed larger than that of metacirculants. Nevertheless, at least for the purposes of this paper it proves natural to work in the context of weak metacirculants.

Below we give a few infinite families of weak metacirculants that will play a crucial role in the investigation of quartic half-arc-transitive metacirculants, the main theme of this article.

Example 2.1 For each $m \geq 3$, for each odd $n \geq 3$ and for each $r \in \mathbb{Z}_{n}^{*}$, where $r^{m}= \pm 1$, let $\mathcal{X}_{o}(m, n ; r)$ be the graph with vertex set $V=\left\{u_{i}^{j} \mid i \in \mathbb{Z}_{m}, j \in \mathbb{Z}_{n}\right\}$ and edges defined by the following adjacencies:

$$
u_{i}^{j} \sim u_{i+1}^{j \pm r^{i}} ; \quad i \in \mathbb{Z}_{m}, j \in \mathbb{Z}_{n} .
$$


(Note that the subscript $o$ in the symbol $\mathcal{X}_{o}(m, n ; r)$ is meant to indicate that $n$ is an odd integer.) The permutations $\rho$ and $\sigma$, defined by the rules

$$
\begin{aligned}
& u_{i}^{j} \rho=u_{i}^{j+1} ; i \in \mathbb{Z}_{m}, j \in \mathbb{Z}_{n} \\
& u_{i}^{j} \sigma=u_{i+1}^{r j} ; i \in \mathbb{Z}_{m}, j \in \mathbb{Z}_{n},
\end{aligned}
$$

are automorphisms of $\mathcal{X}_{o}(m, n ; r)$. Note that $\rho$ is $(m, n)$-semiregular and that $\sigma^{-1} \rho \sigma=\rho^{r}$. Moreover, $\sigma$ cyclically permutes the orbits of $\rho$ and $\sigma^{m}$ fixes $u_{i}^{0}$ for every $i \in \mathbb{Z}_{m}$. Hence $\mathcal{X}_{o}(m, n ; r)$ is an $(m, n)$-metacirculant. We note that graphs $\mathcal{X}_{o}(m, n ; r)$ correspond to the graphs $X(r ; m, n)$ introduced in [20]. We also note that the Holt graph, the smallest half-arc-transitive graph (see $[3,10,15])$, is isomorphic to $\mathcal{X}_{o}(3,9 ; 2)$.

Example 2.2 For each $m \geq 4$ even, $n \geq 4$ even, $r \in \mathbb{Z}_{n}^{*}$, where $r^{m}=1$, and $t \in \mathbb{Z}_{n}$, where $t(r-1)=0$, let $\mathcal{X}_{e}(m, n ; r, t)$ be the graph with vertex set $V=\left\{u_{i}^{j} \mid i \in\right.$ $\left.\mathbb{Z}_{m}, j \in \mathbb{Z}_{n}\right\}$ and edges defined by the following adjacencies:

$$
u_{i}^{j} \sim \begin{cases}u_{i+1}^{j}, u_{i+1}^{j+r^{i}} ; & i \in \mathbb{Z}_{m} \backslash\{m-1\}, j \in \mathbb{Z}_{n} \\ u_{0}^{j+t}, u_{0}^{j+r^{m-1}+t} ; & i=m-1, j \in \mathbb{Z}_{n} .\end{cases}
$$

(In analogy with Example 2.1 the subscript $e$ in the symbol $\mathcal{X}_{e}(m, n ; r, t)$ is meant to indicate that $n$ is an even integer.) The permutations $\rho$ and $\sigma$, defined by the rules

$$
\begin{gathered}
u_{i}^{j} \rho=u_{i}^{j+1} ; \quad i \in \mathbb{Z}_{m}, j \in \mathbb{Z}_{n} \\
u_{i}^{j} \sigma=\left\{\begin{array}{l}
u_{i+1}^{r j} ; i \in \mathbb{Z}_{m} \backslash\{m-1\}, j \in \mathbb{Z}_{n} \\
u_{0}^{r j+t} ; i=m-1, j \in \mathbb{Z}_{n},
\end{array}\right.
\end{gathered}
$$

are automorphisms of $\mathcal{X}_{e}(m, n ; r, t)$. Note that $\rho$ is $(m, n)$-semiregular, that $\sigma^{-1} \rho \sigma=$ $\rho^{r}$ and that $\sigma$ cyclically permutes the orbits of $\rho$. Hence $\mathcal{X}_{e}(m, n ; r, t)$ is a weak $(m, n)$-metacirculant.

As noted in Section 1, a complete classification of quartic tightly attached halfarc-transitive graphs is given in [20] for odd radius and in [29] for even radius. It follows by this classification that a quartic tightly attached half-arc-transitive graph is isomorphic either to some $\mathcal{X}_{o}(m, n ; r)$ or to some $\mathcal{X}_{e}(m, n ; r, t)$, depending on the radius parity.

Example 2.3 For each $m \geq 3, n \geq 3, r \in \mathbb{Z}_{n}^{*}$, where $r^{m}=1$, and $t \in \mathbb{Z}_{n}$ satisfying $t(r-1)=0$, let $\mathcal{Y}(m, n ; r, t)$ be the graph with vertex set $V=\left\{u_{i}^{j} \mid i \in \mathbb{Z}_{m}, j \in \mathbb{Z}_{n}\right\}$ 
and edges defined by the following adjacencies:

$$
u_{i}^{j} \sim\left\{\begin{array}{l}
u_{i}^{j+r^{i}}, u_{i+1}^{j} ; \quad i \in \mathbb{Z}_{m} \backslash\{m-1\}, j \in \mathbb{Z}_{n} \\
u_{m-1}^{j+r^{m-1}}, u_{0}^{j+t} ; i=m-1, j \in \mathbb{Z}_{n} .
\end{array}\right.
$$

The permutations $\rho$ and $\sigma$, defined by the rules

$$
\begin{gathered}
u_{i}^{j} \rho=u_{i}^{j+1} ; \quad i \in \mathbb{Z}_{m}, j \in \mathbb{Z}_{n} \\
u_{i}^{j} \sigma=\left\{\begin{array}{l}
u_{i+1}^{r j} ; i \in \mathbb{Z}_{m} \backslash\{m-1\}, j \in \mathbb{Z}_{n} \\
u_{0}^{r j+t} ; i=m-1, j \in \mathbb{Z}_{n},
\end{array}\right.
\end{gathered}
$$

are automorphisms of $\mathcal{Y}(m, n ; r, t)$. Observe that $\rho$ is $(m, n)$-semiregular and that $\sigma^{-1} \rho \sigma=\rho^{r}$. Moreover, $\sigma$ cyclically permutes the orbits of $\rho$, and so $\mathcal{Y}(m, n ; r, t)$ is a weak $(m, n)$-metacirculant. We note that the Holt graph, see Example 2.1, is also isomorphic to $\mathcal{Y}(3,9 ; 7,3)$.

Example 2.4 For each $m \geq 5, n \geq 3, k \in \mathbb{Z}_{m} \backslash\{0,1,-1\}$ and $r \in \mathbb{Z}_{n}^{*}$, where $r^{m}=1$, let $\mathcal{Z}(m, n ; k, r)$ be the graph with vertex set $V=\left\{u_{i}^{j} \mid i \in \mathbb{Z}_{m}, j \in \mathbb{Z}_{n}\right\}$ and edges defined by the following adjacencies:

$$
u_{i}^{j} \sim u_{i+1}^{j}, u_{i+k}^{j+r^{i}} ; \quad i \in \mathbb{Z}_{m}, j \in \mathbb{Z}_{n} .
$$

The permutations $\rho$ and $\sigma$, defined by the rules

$$
\begin{gathered}
u_{i}^{j} \rho=u_{i}^{j+1} ; \quad i \in \mathbb{Z}_{m}, j \in \mathbb{Z}_{n} \\
u_{i}^{j} \sigma=u_{i+1}^{r j} ; \quad i \in \mathbb{Z}_{m}, j \in \mathbb{Z}_{n},
\end{gathered}
$$

are automorphisms of $\mathcal{Z}(m, n ; k, r)$. Observe that $\rho$ is $(m, n)$-semiregular and that $\sigma^{-1} \rho \sigma=\rho^{r}$. Moreover, $\sigma$ cyclically permutes the orbits of $\rho$ and $\sigma^{m}=1$. Hence $\mathcal{Z}(m, n ; k, r)$ is an $(m, n)$-metacirculant.

\section{The four classes}

In this section we start our investigation of half-arc-transitivity of quartic weak metacirculants. First, we state a result from [20] which will be used throughout the rest of the paper.

Proposition 3.1 [20, Proposition 2.1] Let X be a half-arc-transitive graph. Then no automorphism of $X$ can interchange a pair of adjacent vertices in $X$. 
Throughout this section we let $X$ denote a connected quartic half-arc-transitive weak $(m, n)$-metacirculant relative to an ordered pair $(\rho, \sigma)$. Furthermore, we let $X_{i}, i \in \mathbb{Z}_{m}$, denote the orbits of $\rho$ where $X_{i+1}=X_{i} \sigma$ for each $i \in \mathbb{Z}_{m}$. Clearly, the degrees of subgraphs $\left[X_{i}\right]$ are all equal. We shall denote this number by $d_{i n n}(X)$ and call it the inner degree of $X$. Note that $d_{i n n}(X)$ must be even, for otherwise $n$ is even and a vertex $u$ of $X$ is necessarily adjacent to $u \rho^{\frac{n}{2}}$. But then $\rho^{\frac{n}{2}}$ interchanges two adjacent vertices, which contradicts Proposition 3.1. Furthermore, $d_{i n n}(X)$ cannot be 4 , for otherwise the connectedness of $X$ implies that $m=1$ and thus $X$ is a circulant. But no half-arc-transitive Cayley graph of an abelian group exists. Namely, if $X=$ Cay $(G, S)$ choose $s \in S$, let $\varphi: X \rightarrow X$ map $x$ to $x^{-1}$ and let $\rho_{s}: X \rightarrow X$ map $x$ to $s x$. Then $\varphi$ and $\rho_{s}$ are automorphisms of $X$ and $\varphi \rho_{s}$ interchanges adjacent vertices 1 and $s$. Therefore

$$
d_{i n n}(X) \in\{0,2\} .
$$

We now show that the number of orbits of $\rho$ is at least 3 .

Proposition 3.2 Let $X$ be a connected quartic half-arc-transitive weak $(m, n)$-metacirculant relative to an ordered pair $(\rho, \sigma)$. Then $m \geq 3$.

PROOF: By the above remarks we have $d_{i n n}(X) \in\{0,2\}$ and $m \geq 2$. Assume then that $m=2$ and let $U$ and $W$ be the orbits of $\rho$. We show that there exists an automorphism of $X$ fixing $U$ and $W$ setwise and interchanging two adjacent vertices of $U$ which contradicts Proposition 3.1. By [20, Proposition 2.2.], which states that a graph cannot be half-arc-transitive if it has a $(2, n)$-semiregular automorphism whose two orbits give rise to a bipartition of the graph in question, we must have $d_{i n n}(X)=2$. Fix a vertex $u \in U$ and set $u^{i}=u \rho^{i}$, where $i \in \mathbb{Z}_{n}$. There exists some nonzero $s \in \mathbb{Z}_{n}$ such that $u^{i} \sim u^{i \pm s}$ for all $i \in \mathbb{Z}_{n}$. Next, choose a vertex $w \in W$ such that $u^{0} \sim w$ and set $w^{i}=w \rho^{i}$, where $i \in \mathbb{Z}_{n}$. Letting $r \in \mathbb{Z}_{n}^{*}$ be as in equation (1) we have $u^{0} \sigma \sim$ $u^{ \pm s} \sigma=u^{0} \rho^{ \pm s} \sigma=u^{0} \sigma \rho^{ \pm r s}$, and so $w^{i} \sim w^{i \pm r s}$ for all $i \in \mathbb{Z}_{n}$. There exists some nonzero $t \in \mathbb{Z}_{n}$ such that $u^{0} \sim w^{t}$. Therefore, we have $u^{i} \sim w^{i}, w^{i+t}$ for all $i \in \mathbb{Z}_{n}$. It is easy to see that the permutation $\varphi$ of $V(X)$ defined by the rule $u^{i} \varphi=u^{-i}$ and $w^{i} \varphi=w^{t-i}$, where $i \in \mathbb{Z}_{n}$, is an automorphism of $X$. But then $\varphi \rho^{s}$ interchanges adjacent vertices $u^{0}$ and $u^{s}$, completing the proof of Proposition 3.2.

We now use (2) and Proposition 3.2 to show that each connected quartic half-arctransitive weak metacirculant belongs to at least one of the following four classes reflecting four essentially different ways in which a quartic graph may be a half-arctransitive weak metacirculant (see Figure 1). These four classes are described below. (Recall that the orbits of $\rho$ are denoted by $X_{i}$.)

- Class I. The graph $X$ belongs to Class $I$ if $d_{i n n}(X)=0$ and each orbit $X_{i}$ is connected (with a double edge) to two other orbits. In view of connectedness of $X$, we have that $X_{\rho}$ is a "double-edge" cycle.

- Class II. The graph $X$ belongs to Class II if $d_{i n n}(X)=2$ and each orbit $X_{i}$ is connected (with a single edge) to two other orbits. In view of connectedness of $X$, we have that $X_{\rho}$ is a cycle (with a loop at each vertex). 
Fig. 1 Every quartic half-arc-transitive metacirculant falls into one (or more) of the four classes.

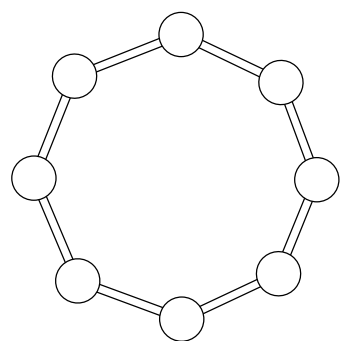

Class I

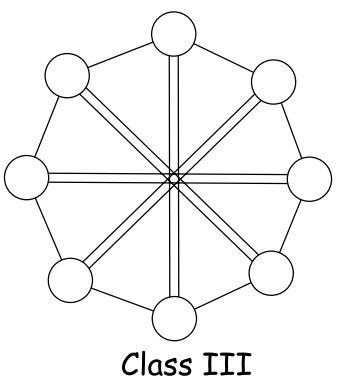

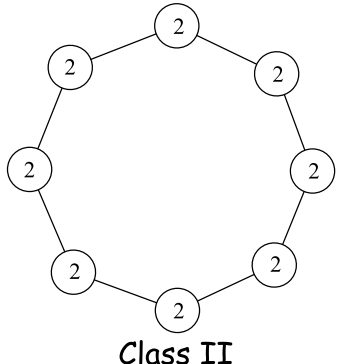

Class II

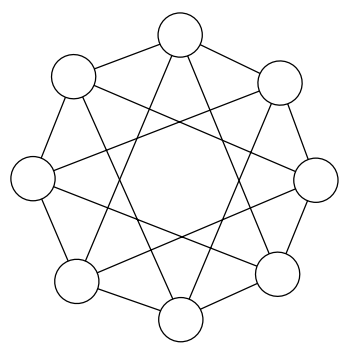

Class IV

- Class III. The graph $X$ belongs to Class III if $d_{i n n}(X)=0$ and each orbit $X_{i}$ is connected to three other orbits, to one with a double edge and to two with a single edge. Clearly, $m$ must be even in this case and an orbit $X_{i}$ is connected to the orbit $X_{i+\frac{m}{2}}$ with a double edge. In short, $X_{\rho}$ is a connected circulant with double edges connecting antipodal vertices.

- Class IV. The graph $X$ belongs to Class $I V$ if $d_{i n n}(X)=0$ and each orbit $X_{i}$ is connected (with a single edge) to four other orbits. In short, $X_{\rho}$ is a connected circulant of valency 4 and is a simple graph.

We remark that these four classes of metacirculants are not disjoint. For instance, it may be seen that the Holt graph $\mathcal{X}_{o}(3,9 ; 2) \cong \mathcal{Y}(3,9 ; 7,3)$ belongs to Classes I and II but not to Classes III and IV. Its canonical double cover, the smallest example in the Bouwer's construction, belongs to Classes I, II and III but not to Class IV. On the other hand, the graph $\mathcal{Z}(20,5 ; 9,2)$ belongs solely to Class IV.

In the next two sections Classes I and II are analyzed in detail. In the last section future research directions regarding interconnectedness of Classes I, II, III and IV, are layed out.

\section{Graphs of Class I}

The aim of this section is to prove the following theorem.

Theorem 4.1 Connected quartic half-arc-transitive weak metacirculants of Class I coincide with connected quartic tightly attached half-arc-transitive graphs. 
Throughout this section we let $X$ denote a connected quartic half-arc-transitive weak metacirculant of Class I and we let $m, n, \rho$ and $\sigma$ be such that $X$ is a weak $(m, n)$-metacirculant relative to the ordered pair $(\rho, \sigma)$. Fix a vertex $u \in V(X)$ and let $u_{i}^{0}=u \sigma^{i}$ for all $i \in\{0,1, \ldots, m-1\}$. Then let $u_{i}^{j}=u_{i}^{0} \rho^{j}$ for all $i \in \mathbb{Z}_{m}, j \in \mathbb{Z}_{n}$. With this notation the orbits of $\rho$ are precisely the sets $X_{i}=\left\{u_{i}^{j} \mid j \in \mathbb{Z}_{n}\right\}, i \in \mathbb{Z}_{m}$. Since $X$ is connected we can assume that $X_{0} \sim X_{1}$ in the quotient graph $X_{\rho}$. Moreover, as $X$ is a weak $(m, n)$-metacirculant relative to the pair $\left(\rho, \sigma \rho^{j}\right)$ for any $j \in \mathbb{Z}_{n}$, we can in fact assume that $u_{0}^{0}$ is adjacent to $u_{1}^{0}$. There exists some nonzero $a \in \mathbb{Z}_{n}$ such that $u_{0}^{0}$ is adjacent also to $u_{1}^{a}$. Therefore $N\left(u_{0}^{0}\right) \cap X_{1}=\left\{u_{1}^{0}, u_{1}^{a}\right\}$. Let $r \in \mathbb{Z}_{n}^{*}$ be as in equation (1). Then $u_{1}^{a} \sigma^{i}=u_{1}^{0} \rho^{a} \sigma^{i}=u_{1}^{0} \sigma^{i} \rho^{r^{i} a}$ holds for all $i \in \mathbb{Z}_{m}$, and so

$$
N\left(u_{i}^{j}\right) \cap X_{i+1}=\left\{u_{i+1}^{j}, u_{i+1}^{j+r^{i} a}\right\} \quad \text { for all } i \in Z_{m} \backslash\{m-1\}, j \in \mathbb{Z}_{n} .
$$

Out of the two orientations of the edges of $X$ induced by the half-arc-transitive action of Aut $X$ we choose the one where $u_{0}^{0} \rightarrow u_{1}^{0}$. Denote the corresponding oriented graph by $D_{X}$. There are two possibilities depending on whether $u_{0}^{0}$ is the tail or the head of the edge $u_{0}^{0} u_{1}^{a}$ in $D_{X}$. In Lemma 4.2 below we show that in the former case $X$ is tightly attached, and in Lemma 4.3 we show that the latter actually never occurs.

Lemma 4.2 With the notation introduced in the previous paragraph, if $u_{0}^{0}$ is the tail of the edge $u_{0}^{0} u_{1}^{a}$ in $D_{X}$ then $X$ is tightly attached.

Proof: Clearly in this case all the edges in $D_{X}\left[X_{i}, X_{i+1}\right]$ are oriented from $X_{i}$ to $X_{i+1}$. Therefore (3) implies that $u_{0}^{j} \rightarrow u_{1}^{j}, u_{1}^{j+a}$ and that $u_{1}^{j} \rightarrow u_{2}^{j}, u_{2}^{j+r a}$ for all $j \in \mathbb{Z}_{n}$. Moreover, any alternating cycle of $X$ is a subgraph of $X\left[X_{i}, X_{i+1}\right]$ for some $i \in \mathbb{Z}_{m}$. We now inspect the two alternating cycles containing $u_{1}^{0}$. Denote the one containing vertices from $X_{0}$ and $X_{1}$ with $C_{1}$ and the one containing vertices from $X_{1}$ and $X_{2}$ with $C_{2}$. In view of Proposition 3.2 we have $m \geq 3$, and so $C_{1} \cap C_{2} \subseteq X_{1}$. We have $C_{1} \cap X_{1}=\left\{u_{1}^{j} \mid j \in\langle a\rangle\right\}$. (Here $\langle a\rangle$ denotes the additive subgroup of $\mathbb{Z}_{n}$ generated by $a$.) Moreover, $C_{2} \cap X_{1}=\left\{u_{1}^{j} \mid j \in\langle r a\rangle\right\}$. But $r \in \mathbb{Z}_{n}^{*}$ and so $\langle a\rangle=\langle r a\rangle$. Hence $C_{1} \cap X_{1}=C_{2} \cap X_{2}$, which completes the proof.

Lemma 4.3 There exists no connected quartic half-arc-transitive weak metacirculant of Class I such that, with the notation from the paragraph preceding the statement of Lemma 4.2, the vertex $u_{0}^{0}$ is the head of the edge $u_{0}^{0} u_{1}^{a}$ in $D_{X}$.

PROOF: Suppose that there does exist such a graph and denote it by $X$. Our approach is as follows. We first show that the stabilizer of a vertex in $X$ cannot be $\mathbb{Z}_{2}$. We then show that this forces $m$ to be odd and $n \equiv 2(\bmod 4)$, which enables us to investigate the Aut $X$-orbit of the so called generic 8-cycles of $X$ in a greater detail. In particular we find that $(r-1)^{2}=0$. Then, investigating the Aut $X$-orbit of a particular nongeneric 8 -cycle, we finally arrive at a contradiction, thus showing that $X$ cannot exist.

Observe that since $\sigma^{m}$ fixes the orbits $X_{i}$ setwise, there exists some $t \in \mathbb{Z}_{n}$, such that $\sigma^{m} \rho^{-t}$ fixes $u_{0}^{0}$. Since the sets $X_{i}$ are blocks of imprimitivity for $\langle\rho, \sigma\rangle$, the 
particular orientation of the edges in $D_{X}$ implies that $\sigma^{m} \rho^{-t}$ fixes the neighbors of $u_{0}^{0}$ pointwise. Continuing this way we have, in view of connectedness of $X$, that $\sigma^{m}=\rho^{t}$. Note that equation (1) implies that $\sigma^{-m} \rho \sigma^{m}=\rho^{r^{m}}$, and so

$$
r^{m}=1
$$

Moreover, (1) also implies that $u_{i}^{j} \sigma=u_{i}^{0} \rho^{j} \sigma=u_{i}^{0} \sigma \rho^{r j}$, and so

$$
u_{i}^{j} \sigma=\left\{\begin{array}{l}
u_{i+1}^{r j} ; i \in \mathbb{Z}_{m} \backslash\{m-1\}, j \in \mathbb{Z}_{n} \\
u_{0}^{r j+t} ; i=m-1, j \in \mathbb{Z}_{n} .
\end{array}\right.
$$

Combining together (3) and (5), we have that for any $i \in \mathbb{Z}_{m}$ and $j \in \mathbb{Z}_{n}$, the two edges connecting $u_{i}^{j}$ to vertices from $X_{i+1}$ in $D_{X}$ are given by

$$
u_{i}^{j} \rightarrow\left\{\begin{array} { l } 
{ u _ { i + 1 } ^ { j } ; i \neq m - 1 } \\
{ u _ { 0 } ^ { j + t } ; i = m - 1 }
\end{array} \text { and } \quad u _ { i } ^ { j } \leftarrow \left\{\begin{array}{ll}
u_{i+1}^{j+r^{i} a} ; & i \neq m-1 \\
u_{0}^{j+r^{m-1} a+t} ; i=m-1
\end{array}\right.\right.
$$

Since $\sigma$ maps the edge $u_{m-1}^{0} u_{0}^{t}$ to the edge $u_{0}^{t} u_{1}^{r t}$, we also have

$$
t(r-1)=0 .
$$

CLAIM 1: We lose no generality in assuming that $a=1$.

Observe that since $X$ is connected, (6) implies that $\langle a, t\rangle=\mathbb{Z}_{n}$. Let $d=|a|$ denote the order of $a$ in the additive group $\mathbb{Z}_{n}$. Clearly Claim 1 holds if $d=n$, so assume that $d<n$ and set $k=\frac{n}{d}$. Let $\rho^{\prime}=\rho^{a}$. Then $\rho^{\prime}$ is an $(m k, d)$-semiregular automorphism of $X$ and $\sigma^{-1} \rho^{\prime} \sigma=\rho^{r a}=\rho^{\prime r}$. We now show that $\sigma$ cyclically permutes the orbits of $\rho^{\prime}$. The orbit of $\rho^{\prime}$ containing $u_{0}^{0}$ is $X_{0}^{\prime}=\left\{u_{0}^{j a} \mid j \in \mathbb{Z}_{n}\right\}$. Moreover, $X_{i}^{\prime}=X_{0}^{\prime} \sigma^{i}=$ $\left\{u_{i}^{j r^{i} a} \mid j \in \mathbb{Z}_{n}\right\}$ for $i=0,1, \ldots, m-1$ and $X_{m}^{\prime}=X_{0}^{\prime} \sigma^{m}=\left\{u_{0}^{j a+t} \mid j \in \mathbb{Z}_{n}\right\}$. Since $\langle a, t\rangle=\mathbb{Z}_{n}$ and $\langle a\rangle \neq \mathbb{Z}_{n}$, the set $X_{m}^{\prime}$ (which is clearly an orbit of $\rho^{\prime}$ ) cannot be equal to $X_{0}^{\prime}$. Continuing this way we see that $\sigma$ cyclically permutes the $m k$ orbits of $\rho^{\prime}$. Thus $X$ is a weak $(m k, d)$-metacirculant of Class I relative to the ordered pair $\left(\rho^{\prime}, \sigma\right)$. It is now clear that in the notation of vertices of $X$ relative to the ordered pair $\left(\rho^{\prime}, \sigma\right)$ the corresponding parameter $a^{\prime}$ is equal to 1 . From now on we can therefore assume that $a=1$.

Claim 2: Let $v \in V(X)$. Then $\left|(\operatorname{Aut} X)_{v}\right|>2$.

Suppose on the contrary that (Aut $X)_{v} \cong \mathbb{Z}_{2}$ for some (and hence any) $v \in V(X)$. Let $\tau$ be the unique nontrivial automorphism of (Aut $X)_{u_{0}^{0}}$. Then $\tau$ interchanges $u_{1}^{0}$ and $u_{m-1}^{-r^{m-1}-t}$ and also interchanges $u_{1}^{1}$ and $u_{m-1}^{-t}$. We now determine the action of $\tau$ on the vertices of $X$ recursively as follows. Since $\tau \notin\langle\rho, \sigma\rangle$ and since $u_{1}^{1} \tau=u_{m-1}^{-t}=u_{1}^{1} \sigma^{m-2} \rho^{-r^{m-2}-t}$ we have (recall that (Aut $\left.X\right)_{u_{1}^{1}} \cong \mathbb{Z}_{2}$ ) that $u_{0}^{1} \tau \neq$ 
$u_{0}^{1} \sigma^{m-2} \rho^{-r^{m-2}-t}=u_{m-2}^{-t}$. It follows that $\tau$ interchanges $u_{0}^{1}$ and $u_{0}^{r^{m-1}}$. Now $u_{0}^{1} \leftarrow u_{1}^{2}$ and a similar argument shows that $\tau$ interchanges $u_{1}^{2}$ and $u_{m-1}^{r^{m-1}-t}$. Continuing this way we find that $\tau$ maps according to the rule:

$$
u_{i}^{j} \tau= \begin{cases}u_{m-i}^{j r^{m-1}-r^{m-1}-r^{m-2}-\cdots-r^{m-i}-t} & ; i \in \mathbb{Z}_{m} \backslash\{0\}, j \in \mathbb{Z}_{n} \\ u_{0}^{j r^{m-1}} ; & i=0, j \in \mathbb{Z}_{n} .\end{cases}
$$

We leave the details to the reader. Recall now that, by assumption, $\tau$ interchanges $u_{m-1}^{-t}$ and $u_{1}^{1}$. On the other hand, (8) implies that $u_{m-1}^{-t} \tau=u_{1}^{-t r^{m-1}-r^{m-1}-r^{m-2}-\cdots-r-t}$. Therefore, equation (7) implies that

$$
1+r+r^{2}+\cdots+r^{m-1}+2 t=0
$$

We now define a mapping $\psi$ on $V(X)$ by the rule

$$
u_{i}^{j} \psi= \begin{cases}u_{i}^{-j+1+r+r^{2}+\cdots+r^{i-1}} ; & ; \in \mathbb{Z}_{m} \backslash\{0\}, j \in \mathbb{Z}_{n} \\ u_{0}^{-j} ; & i=0, j \in \mathbb{Z}_{n} .\end{cases}
$$

Clearly $\psi$ is a bijection. It is easy to check that $\psi$ maps every edge of $\left[X_{i}, X_{i+1}\right]$, where $i \in \mathbb{Z}_{m} \backslash\{m-1\}$, to an edge of $X$. As for the edges of $\left[X_{m-1}, X_{0}\right]$, note that by (6) we have that $N\left(u_{m-1}^{j}\right) \cap X_{0}=\left\{u_{0}^{j+t}, u_{0}^{j+r^{m-1}+t}\right\}$. Observe that $\psi$ maps the latter two vertices to $u_{0}^{-j-t}$ and $u_{0}^{-j-r^{m-1}-t}$, respectively. Moreover, in view of equation (9) we have that $u_{m-1}^{j} \psi=u_{m-1}^{-j+1+r+r^{2}+\cdots+r^{m-2}}=u_{m-1}^{-j-r^{m-1}-2 t}$, and so $\psi$ is an automorphism of $X$. Since $X$ is half-arc-transitive, there exists some $\varphi \in \operatorname{Aut} X$ mapping the edge $u_{1}^{1} u_{0}^{0}$ of $D_{X}$ to the edge $u_{0}^{0} u_{1}^{0}$. But then $\psi \varphi$ interchanges adjacent vertices $u_{0}^{0}$ and $u_{1}^{0}$, contradicting Proposition 3.1. Therefore, $\mid(\text { Aut } X)_{v} \mid>2$, as claimed.

Let now $A$ be the attachment set of $X$ containing $u_{0}^{0}$. In view of [27, Lemma 3.5.], which states that in a finite connected quartic half-arc-transitive graph with attachment sets containing at least three vertices, the vertex stabilizers are isomorphic to $\mathbb{Z}_{2}$, it follows that $|A| \leq 2$. We now show that $m$ must be odd.

Claim 3: $m$ is odd.

Suppose on the contrary that $m$ is even. Consider the alternating cycle containing the edge $u_{0}^{0} u_{1}^{0}$. It contains vertices $u_{2}^{r}, u_{3}^{r}, u_{4}^{r+r 3}, \ldots, u_{m-1}^{r+r^{3}+\cdots+r^{m-3}}, u_{0}^{r+r^{3}+\cdots+r^{m-1}+t}$, etc., where $u_{0}^{r+r^{3}+\cdots+r^{m-1}+t}$ is the tail of the two corresponding incident edges on this cycle. The other alternating cycle containing $u_{0}^{0}$ contains vertices $u_{1}^{1}, u_{2}^{1}, u_{3}^{1+r^{2}}, u_{4}^{1+r^{2}}$, $\ldots, u_{m-1}^{1+r^{2}+\cdots+r^{m-2}}, u_{0}^{1+r^{2}+\cdots+r^{m-2}+t}$, etc., where $u_{0}^{1+r^{2}+\cdots+r^{m-2}+t}$ is the head of the two corresponding incident edges on this cycle. Observe that equation (7) implies that $r\left(1+r^{2}+\cdots+r^{m-2}+t\right)=r+r^{3}+\cdots+r^{m-1}+t$, and so the vertices 
$u_{0}^{r+r^{3}+\cdots+r^{m-1}+t}$ and $u_{0}^{1+r^{2}+\cdots+r^{m-2}+t}$ are both contained in $A$. Since $|A|=2$, it follows that $1+r^{2}+\cdots+r^{m-2}+t=r+r^{3}+\cdots+r^{m-1}+t$ and in addition either $1+r^{2}+\cdots+r^{m-2}+t=0$ or $1+r^{2}+\cdots+r^{m-2}+t=\frac{n}{2}$ with $n$ even. But in both cases equation (9) holds, and so the mapping $\psi$ defined as in (10) is an automorphism of $X$ which is impossible. Therefore, $m$ is odd, as claimed.

CLAIM $4: n \equiv 2(\bmod 4)$.

Let $C_{1}$ denote the alternating cycle containing the edge $u_{0}^{0} u_{1}^{0}$. Since $m$ is odd, the vertices $u_{0}^{r+r^{3}+\cdots+r^{m-2}+t}$ and $u_{0}^{1+r+r^{2}+\cdots+r^{m-1}+2 t}$ are both contained in $C_{1}$ with $u_{0}^{r+r^{3}+\cdots+r^{m-2}+t}$ being the head of the two corresponding incident edges on $C_{1}$. Let $C_{2}$ denote the other alternating cycle containing the vertex $u_{0}^{0}$. Then $u_{0}^{1+r^{2}+\cdots+r^{m-1}+t}$ and $u_{0}^{1+r+r^{2}+\cdots+r^{m-1}+2 t}$ are vertices of $C_{2}$ with $u_{0}^{1+r^{2}+\cdots+r^{m-1}+t}$ being the tail of the two corresponding incident edges on $C_{2}$. Since $|A| \leq 2$, we thus have that $1+r+r^{2}+$ $\cdots+r^{m-1}+2 t$ is equal either to 0 or to $\frac{n}{2}$, where in the latter case $n$ must be even. As the former contradicts half-arc-transitivity of $X$ (see the argument immediately after equation (9)), we have that $n$ is even and that

$$
1+r+r^{2}+\cdots+r^{m-1}+2 t=\frac{n}{2} .
$$

Since $r \in \mathbb{Z}_{n}^{*}, r$ is odd. But this implies that $1+r+\left(r^{2}+r^{3}\right)+\cdots+\left(r^{m-3}+r^{m-2}\right)+$ $r^{m-1}+2 t$ is odd too, and so equation (11) implies that $n \equiv 2(\bmod 4)$, as claimed.

CLAIM 5: $C_{0}=u_{0}^{0} u_{1}^{0} u_{2}^{r} u_{1}^{r} u_{0}^{r} u_{1}^{1+r} u_{2}^{1+r} u_{1}^{1}$ is an 8-cycle of $X$.

We only need to see that the cardinality of the set $\{0,1, r, r+1\}$ is 4 , that is, we need to see that $r \neq \pm 1$. Note first that $r \neq 1$ for otherwise $X$ would be a Cayley graph of an abelian group and thus arc-transitive. Furthermore, $r \neq-1$ for then $r^{m}=$ $-1 \neq 1$, contradicting (4). (Note that $n \neq 2$ for otherwise $X$ would be isomorphic to a lexicographic product of a cycle and $2 K_{1}$, and thus clearly arc-transitive.)

The 8-cycles belonging to the $\langle\rho, \sigma\rangle$-orbit of $C_{0}$ will be called the generic 8 -cycles of $X$. We now investigate which 8-cycles, apart from the generic ones, are contained in the Aut $X$-orbit of $C_{0}$. We assume first that $m \geq 5$, as in this case, since $m$ is odd, no 8-cycle containing edges from every subgraph $\left[X_{i}, X_{i+1}\right], i \in \mathbb{Z}_{m}$, exists.

By Claim 2 there exists an automorphism $\varphi \in \operatorname{Aut} X$, fixing $u_{1}^{1}$ and $u_{0}^{0}$ but interchanging $u_{2}^{1+r}$ and $u_{0}^{1}$. We either have $u_{1}^{0} \varphi=u_{1}^{0}$ or $u_{1}^{0} \varphi=u_{m-1}^{-r^{m-1}-t}$. Suppose first that $\varphi$ fixes $u_{1}^{0}$. Then it also fixes $u_{2}^{r}$. Now since $C_{0} \varphi$ is an 8-cycle and since $u_{1}^{1+r}$ is the tail of both of its incident edges on $C_{0}$, we must have $u_{1}^{1+r} \varphi=u_{1}^{2}$. Therefore, $u_{0}^{r} \varphi=u_{2}^{2}$. This leaves us with two possibilities for $u_{1}^{r} \varphi$. If $u_{1}^{r} \varphi=u_{1}^{2-r}$, then $u_{1}^{2-r} \rightarrow u_{2}^{r}$, and so $2(r-1)=0$. But $r$ is odd, so that Claim 4 implies $r-1=0$, a contradiction. Thus $u_{1}^{r} \varphi=u_{3}^{2}$ and so $u_{2}^{r} \leftarrow u_{3}^{2}$, which forces

$$
2-r-r^{2}=0
$$


Suppose now that $u_{1}^{0} \varphi=u_{m-1}^{-r^{m-1}-t}$. It follows that $u_{2}^{r} \varphi=u_{m-2}^{-r^{m-1}-t}$ and then a similar argument as above shows that $u_{1}^{1+r} \varphi=u_{m-1}^{1-t}$, and so $u_{0}^{r} \varphi=u_{m-2}^{1-r^{m-2}-t}$. We now have two possibilities. Either $u_{1}^{r} \varphi=u_{m-1}^{1-r^{m-2}-t}$, which by (4) implies that (12) holds, or $u_{1}^{r} \varphi=u_{m-3}^{1-r^{m-3}-r^{m-2}-t}$, in which case $1-r^{m-3}-r^{m-2}-t=-r^{m-1}-t$, that is,

$$
r^{3}+r^{2}=r+1 \text { or equivalently }(r-1)(r+1)^{2}=0 .
$$

We now show that (13) cannot hold. Namely, multiplying by $r^{2(i-1)}$ we get that $r^{2 i+1}+r^{2 i}=r+1$ for every $i \in \mathbb{N}$. Furthermore, by Claim 3 there exists some integer $k$ such that $m=2 k+1$, and so

$$
1+r+r^{2}+\cdots+r^{m-2}+r^{m-1}=k(1+r)+r^{m-1} .
$$

By (4) we therefore have

$$
0=\left(1+r+\cdots+r^{m-1}\right)(r-1)=k\left(r^{2}-1\right)+r^{m-1}(r-1) .
$$

Multiplying by $r$ and using (13) we obtain

$$
0=k\left(r^{3}-r\right)+r-1=k\left(1-r^{2}\right)+r-1=-k(r-1)(r+1)+(r-1) .
$$

Now, since $r-1 \neq 0$ is even, Claim 4 and equation (13) imply that there exists some odd prime $q$ dividing $r+1$ and $n$ but not $r-1$. However, equation (15) implies that $q$ does divide $r-1$, a contradiction. Therefore, (13) cannot hold, and so (12) holds.

Again using Claim 2, there also exists an automorphism $\vartheta \in \operatorname{Aut} X$ fixing $u_{0}^{0}$ and $u_{1}^{0}$ but interchanging $u_{1}^{1}$ and $u_{m-1}^{-t}$. An analysis similar to the one used above shows that the only possibilities for the image $C_{0} \vartheta$ are:

$$
\begin{aligned}
& u_{0}^{0} u_{1}^{0} u_{2}^{r} u_{3}^{r+r^{2}} u_{2}^{r+r^{2}} u_{1}^{r+r^{2}} u_{0}^{-1+r+r^{2}} u_{m-1}^{-t}, \\
& u_{0}^{0} u_{1}^{0} u_{2}^{r} u_{1}^{r} u_{2}^{2 r} u_{1}^{2 r} u_{0}^{-1+2 r} u_{m-1}^{-t} \text { and } \\
& u_{0}^{0} u_{1}^{0} u_{2}^{r} u_{1}^{r} u_{0}^{r} u_{m-1}^{r-t} u_{m-2}^{r-r^{m-2}-t} u_{m-1}^{-t} .
\end{aligned}
$$

The conditions for the above 8-cycles to exist are, respectively, (13),

$$
1+r-2 r^{2}=0
$$

and

$$
r^{3}=1 \text {. }
$$

Recall that (13) cannot hold and that (12) does hold. It is easy to check that (12) and (16) imply (17) and that (12) and (17) imply (16). Thus all three conditions hold. From (12) and (16) we get that

$$
(r-1)^{2}=0 .
$$

Then $C=u_{0}^{0} u_{1}^{0} u_{2}^{r} u_{3}^{r} u_{2}^{r-r^{2}} u_{1}^{r-r^{2}} u_{2}^{2 r-r^{2}} u_{1}^{2 r-r^{2}}=u_{0}^{0} u_{1}^{0} u_{2}^{r} u_{3}^{r} u_{2}^{1-r} u_{1}^{1-r} u_{2}^{1} u_{1}^{1}$ is an 8cycle of $X$ (recall that $r \neq 1$ ). By Claim 2 there exists an automorphism $\eta \in \operatorname{Aut} X$ fixing $u_{2}^{r}$ and $u_{3}^{r}$ but interchanging $u_{2}^{r-r^{2}}$ with $u_{4}^{r}$. But this implies $u_{1}^{0} \eta=u_{1}^{0}, u_{0}^{0} \eta=u_{0}^{0}$ 
and $u_{1}^{r-r^{2}} \eta=u_{5}^{r+r^{4}}$, and so $C \eta$ is not an 8-cycle, a contradiction. This shows that $X$ cannot exist when $m \geq 5$.

Since $m$ is odd this leaves us with $m=3$ as the only other possibility. A similar analysis as in the general case shows that the only possibilities for $C_{0} \varphi$ (where $\varphi \in$ Aut $X$ fixes $u_{1}^{1}$ and $u_{0}^{0}$ but interchanges $u_{2}^{1+r}$ and $u_{0}^{1}$ ) are 8-cycles which exist only when (12) holds. (In this analysis we get that the only possibilities not encountered in the general case are those for which $2 r+r^{2}+2 t=0$ or $2+r^{2}+2 t=0$, which are of course both impossible as $n$ is even.) By (4) we have $r^{3}=1$, and so multiplying by $r$ in (12) we get that (18) holds. Thus the 8-cycle $C=u_{0}^{0} u_{1}^{0} u_{2}^{r} u_{0}^{r+t} u_{2}^{1-r} u_{1}^{1-r} u_{2}^{1} u_{1}^{1}$ exists in $X$. Again let $\eta \in$ Aut $X$ be an automorphism fixing $u_{2}^{r}$ and $u_{0}^{r+t}$ but interchanging $u_{2}^{1-r}$ with $u_{1}^{r+t}$. Then $u_{1}^{0} \eta=u_{1}^{0}, u_{0}^{0} \eta=u_{0}^{0}$ and $u_{1}^{1-r} \eta=u_{2}^{2 r+t}$. Note that we cannot have $u_{1}^{1} \eta=u_{1}^{1}$ for otherwise $u_{2}^{1} \eta=u_{2}^{1}$, which contradicts the fact that $u_{2}^{1} \sim u_{1}^{r-r^{2}}$. It follows that $u_{1}^{1} \eta=u_{2}^{-t}$, and so $u_{2}^{1} \eta=u_{1}^{-r-t}$. Consequently $-r-t=2 r+t$, that is $3 r+2 t=0$, which forces $1+r+r^{2}+2 t=0$. As this contradicts (11), the proof is complete.

We are now ready to prove Theorem 4.1.

\section{PROOF OF THEOREM 4.1:}

That connected quartic half-arc-transitive weak metacirculants of Class I are tightly attached now follows by Lemmas 4.2 and 4.3. To prove the converse observe, as already noted in Section 2 , that the results of [20,29] imply that every connected quartic tightly attached half-arc-transitive graph is isomorphic either to some $\mathcal{X}_{o}(m, n ; r)$ or to some $\mathcal{X}_{e}(m, n ; r, t)$. As these two graphs are clearly both weak metacirculants of Class I, the proof is complete.

\section{Graphs of Class II}

In this section connected quartic half-arc-transitive metacirculants of Class II are studied in great detail. The following theorem is the main result.

Theorem 5.1 Let $X$ be a connected quartic half-arc-transitive weak $(m, n)$-metacirculant of Class II. Then the following hold:

(i) $X$ is a Cayley graph for the group $\langle\rho, \sigma\rangle$, where $(\rho, \sigma)$ is some pair of automorphisms of $X$ such that $X$ is a weak $(m, n)$-metacirculant of Class II relative to $(\rho, \sigma)$,

(ii) $(\text { Aut } X)_{v} \cong \mathbb{Z}_{2}$ for all $v \in V(X)$,

(iii) $m$ divides $n$ and $d_{m}=\frac{n}{m}>2$,

(iv) there exist $r \in \mathbb{Z}_{n}^{*}$ and $t \in \mathbb{Z}_{n}$ such that $X \cong \mathcal{Y}(m, n ; r, t)$, where parameters $r$ and $t$ satisfy the following conditions:

- $r^{m}=1$,

- $m(r-1)=t(r-1)=(r-1)^{2}=0$,

- $\langle m\rangle=\langle t\rangle$ in $\mathbb{Z}_{n}$,

- there exists a unique $c \in\left\{0,1, \ldots, d_{m}-1\right\}$ such that $t=c m$ and $m=c t$ and

- there exists a unique $a \in\left\{0,1, \ldots, d_{m}-1\right\}$ such that at $=-a m=r-1$, 
(v) $X$ is tightly attached unless $m$ and $d_{m}$ are both even, and $n=8 n_{1}$ for some integer $n_{1}>2$, where $n_{1}$ is even or not squarefree.

Then, using this result, we present a list of all connected quartic half-arc-transitive weak metacirculants of Class II of order up to 1000 which are not tightly attached (see Table 3). Finally, we construct an infinite family of such graphs (see Construction 5.10).

Throughout this section we let $X$ denote a connected quartic half-arc-transitive weak $(m, n)$-metacirculant of Class II. Choose some automorphisms $\rho$ and $\sigma$ such that $X$ is a weak $(m, n)$-metacirculant of Class II relative to the ordered pair $(\rho, \sigma)$. Fix a vertex $u \in V(X)$ and let $u_{i}^{0}=u \sigma^{i}$ for all $i \in\{0,1, \ldots, m-1\}$. Then let $u_{i}^{j}=$ $u_{i}^{0} \rho^{j}$ for all $i \in \mathbb{Z}_{m}, j \in \mathbb{Z}_{n}$. Thus $X_{i}=\left\{u_{i}^{j} \mid j \in \mathbb{Z}_{n}\right\}, i \in \mathbb{Z}_{m}$, are the orbits of $\rho$ and $X_{i}=X_{0} \sigma^{i}$. We shall say that an edge connecting vertices from the same orbit $X_{i}$ is an inner edge and that an edge connecting vertices from different orbits is an outer edge.

Since $d_{i n n}(X)=2$, there exists some nonzero $s \in \mathbb{Z}_{n}$ such that $u_{0}^{j} \sim u_{0}^{j \pm s}$ for all $j \in \mathbb{Z}_{n}$. Fix an orientation of edges induced on $X$ by the half-arc-transitive action of Aut $X$ and denote the corresponding directed graph by $D_{X}$. Then the indegrees and the outdegrees of the subgraphs of $D_{X}$ induced by $X_{i}$ are all equal to 1 . We will assume that $u_{0}^{j-s} \rightarrow u_{0}^{j} \rightarrow u_{0}^{j+s}$. Letting $r \in \mathbb{Z}_{n}^{*}$ be as in equation (1), we have that $u_{0}^{s} \sigma^{i}=u_{0}^{0} \rho^{s} \sigma^{i}=u_{0}^{0} \sigma^{i} \rho^{r^{i} s}$, and so

$$
u_{i}^{j-r^{i} s} \rightarrow u_{i}^{j} \rightarrow u_{i}^{j+r^{i} s}, \quad \text { for all } i \in \mathbb{Z}_{m}, j \in \mathbb{Z}_{n}
$$

There exists some $k \in \mathbb{Z}_{m} \backslash\{0\}$ such that the vertices from the orbit $X_{0}$ are adjacent to the vertices from the orbit $X_{k}$. Since $X$ is connected, $\langle k\rangle=\mathbb{Z}_{m}$, so that we can assume $k=1$ (otherwise take $\sigma^{\prime}=\sigma^{k}$ and $r^{\prime}=r^{k}$ ). Let $a \in \mathbb{Z}_{n}$ be such that $u_{0}^{0} \sim u_{1}^{a}$. We can assume that $u_{0}^{0} \rightarrow u_{1}^{a}$ (otherwise take $\rho^{\prime}=\rho^{-1}$ and then choose the other of the two possible orientations of the edges for $D_{X}$ ). With no loss of generality we can also assume that $a=0$ (otherwise take $\sigma^{\prime}=\sigma \rho^{a}$ ). Therefore, $u_{i}^{j} \rightarrow u_{i+1}^{j}$ for all $i \in \mathbb{Z}_{m} \backslash\{m-1\}, j \in \mathbb{Z}_{n}$. Since $\sigma$ cyclically permutes the $m$ orbits of $\rho$, we have that $u_{0}^{0} \sigma^{m} \in X_{0}$. Thus, there exists a unique $t \in \mathbb{Z}_{n}$ such that $u_{0}^{0} \sigma^{m} \rho^{-t}=u_{0}^{0}$. Since the orbits $X_{i}$ are blocks of imprimitivity for the group $H=\langle\rho, \sigma\rangle$, half-arc-transitivity of $X$ and the orientation of the edges of $D_{X}$ imply that an element of $H$ fixing a vertex must necessarily fix all of its neighbors pointwise. By connectedness of $X$ we then have that

the group $\langle\rho, \sigma\rangle$ acts regularly on $V(X)$.

In particular, $\sigma^{m}=\rho^{t}$. This implies that $\rho=\rho^{-t} \rho \rho^{t}=\sigma^{-m} \rho \sigma^{m}=\rho^{r^{m}}$, and so

$$
r^{m}=1
$$


Moreover, $u_{i}^{j} \sigma=u_{i}^{0} \rho^{j} \sigma=u_{i}^{0} \sigma \rho^{r j}=u_{i+1}^{0} \rho^{r j}=u_{i+1}^{r j}$ for $i \neq m-1, j \in \mathbb{Z}_{n}$. By (21) we now also have $u_{m-1}^{j} \sigma=u_{0}^{r j} \sigma^{m-1} \sigma=u_{0}^{r j} \rho^{t}=u_{0}^{r j+t}$, and so

$$
\begin{gathered}
u_{i}^{j} \sigma=\left\{\begin{array}{l}
u_{i+1}^{r j} ; i \in \mathbb{Z}_{m} \backslash\{m-1\}, j \in \mathbb{Z}_{n} \\
u_{0}^{r j+t} ; i=m-1, j \in \mathbb{Z}_{n}
\end{array} \quad\right. \text { and } \\
u_{i}^{j} \rightarrow\left\{\begin{array}{l}
u_{i+1}^{j} ; i \in \mathbb{Z}_{m} \backslash\{m-1\}, j \in \mathbb{Z}_{n} \\
u_{0}^{j+t} ; i=m-1, j \in \mathbb{Z}_{n} .
\end{array}\right.
\end{gathered}
$$

Let us now consider the edge $u_{m-1}^{0} u_{0}^{t}$. By (22), $\sigma$ maps this edge to the edge $u_{0}^{t} u_{1}^{r t}$, and so (23) implies that $r t=t$, that is,

$$
t(r-1)=0 .
$$

We claim that $r s \neq \pm s$. Suppose on the contrary that $r s=s$ or $r s=-s$ and consider the permutation $\varphi$ of $V(X)$ defined by the rule $u_{0}^{j} \varphi=u_{0}^{-j}$, where $j \in \mathbb{Z}_{n}$, and $u_{i}^{j} \varphi=u_{m-i}^{-j-t}$, where $i \in \mathbb{Z}_{m} \backslash\{0\}$ and $j \in \mathbb{Z}_{n}$. Since $r s= \pm s$, we have that either $r^{i} s=s$ or that $r^{i} s=(-1)^{i} s$ for all $i \in \mathbb{Z}_{m}$. It is now easy to check that $\varphi$ is an automorphism of $X$. But $\varphi \rho^{s}$ interchanges adjacent vertices $u_{0}^{0}$ and $u_{0}^{s}$, which by Proposition 3.1 contradicts half-arc-transitivity of $X$.

We now investigate certain 8-cycles of $X$ in order to obtain a better understanding of the structural properties of $X$. Consider the following closed walk of $X$ :

$$
\left(u_{0}^{0}, u_{0}^{s}, u_{1}^{s}, u_{1}^{s+r s}, u_{0}^{s+r s}, u_{0}^{r s}, u_{1}^{r s}, u_{1}^{0}, u_{0}^{0}\right) .
$$

Since $s \neq 0, r \in \mathbb{Z}_{n}^{*}$ and $r s \neq \pm s$, it follows that the above 8 vertices are all distinct, and so the closed walk (25) gives rise to an 8-cycle. Every 8-cycle of $X$ belonging to the $H$-orbit of this 8 -cycle will be called a generic 8 -cycle.

To every 8 -cycle $C$ of $X$ we assign a binary sequence as follows. When traversing $C$, we assign value 1 to each edge of $X$ traversed along its orientation in $D_{X}$, and we assign value 0 to each edge of $X$ traversed against its orientation in $D_{X}$. We say that two binary sequences corresponding to 8 -cycles of $X$ are equivalent if one can be obtained from the other using cyclic rotations and reflections. We let the code of $C$ be the equivalence class of its sequences and we denote it by any of the corresponding sequences. Therefore, the code of the generic 8-cycle given in (25) is 11100100 (see Figure 2). Note that, since $X$ is half-arc-transitive, the code of a cycle is invariant under the action of Aut $X$. On the other hand, there exists an automorphism $\tau \in \operatorname{Aut} X$ fixing $u_{1}^{r s}$ and interchanging $u_{1}^{0}$ and $u_{0}^{r s}$. Since $u_{0}^{r s} \rightarrow u_{0}^{s+r s}$, we thus have that $u_{0}^{s+r s} \tau=u_{2}^{0}$. Consequently, the image under $\tau$ of the generic 8-cycle corresponding to (25) is an 8-cycle consisting of vertices from at least three orbits $X_{i}$ and is therefore not generic. The following lemma gives all possible $H$-orbits of 8 -cycles of $X$ having code 11100100 .

Lemma 5.2 With the notation introduced in this section the only possible $H$-orbits of 8-cycles having code 11100100 in $X$ are given in Table 1, together with the cor- 
responding representatives and the necessary and sufficient arithmetic conditions for their existence.

PROOF: Let $C=c_{0} c_{1} c_{2} c_{3} c_{4} c_{5} c_{6} c_{7}$ be an 8-cycle with code 11100100 . We divide our investigation into several cases depending on the number of orbits $X_{i}$ the 8-cycle $C$ meets.

CASE 1: $C$ meets one orbit.

As any such 8-cycle has code 11111111 , this case cannot occur.

\section{CASE 2: $C$ meets two orbits.}

Clearly, the number of outer edges of $C$ is even. In fact, $C$ either has 2 or 4 outer edges. The former case is impossible for otherwise $C$ contains at least four consecutive vertices in a single orbit and thus 1111 is a subsequence of the code of $C$. It is thus clear that the inner and outer edges alternate on $C$. Therefore, the first and the last 1 of the subsequence 111 of the code of $C$ both correspond to inner edges, and so it is clear that $C$ is a generic 8 -cycle.

CASE 3: $C$ meets three orbits, say, with no loss of generality, $X_{0}, X_{1}$ and $X_{2}$.

Suppose first that $m>3$. Therefore, if $c_{i} \in X_{0}$ or $c_{i} \in X_{2}$, at least one of $c_{i-1}, c_{i+1}$ lies in the same orbit as $c_{i}$. This implies that no four consecutive vertices of $C$ are contained in a single orbit. Namely, they cannot be contained in $X_{0}$ or $X_{2}$, for otherwise the code of $C$ would contain 1111 as a subsequence. Moreover, they cannot be contained in $X_{1}$ since there are no edges between $X_{0}$ and $X_{2}$. We now show that no three consecutive vertices of $C$ are contained in a single orbit. Suppose on the contrary that $c_{0}, c_{1}$ and $c_{2}$ are all contained in one orbit. If this orbit is $X_{0}$, then $c_{3}, c_{7} \in X_{1}$, so in order to have the required code, at least one of $c_{4}$ and $c_{6}$ lies in $X_{1}$. But then the remaining two vertices lie in $X_{2}$, so the code cannot be 11100100 . A similar argument shows that the orbit containing $c_{0}, c_{1}$ and $c_{2}$ cannot be $X_{2}$. Suppose now that the orbit containing $c_{0}, c_{1}$ and $c_{2}$ is $X_{1}$. It is then clear that one of $c_{3}$ and $c_{7}$ lies in $X_{0}$ and the other in $X_{2}$; say $c_{3} \in X_{0}$ and $c_{7} \in X_{2}$. It follows that $c_{4} \in X_{0}, c_{5} \in X_{1}$ and $c_{6} \in X_{2}$. It is easy to see however, that such an 8-cycle does not have code 11100100 . Therefore, no three consecutive vertices of $C$ lie on a single orbit. Consequently, each of $X_{0}$ and $X_{2}$ contains two vertices of $C$, and so four vertices of $C$ are contained in $X_{1}$. As $C$ has code 11100100 , it is now clear that $C$ lies in the $H$-orbit of 8 -cycles from row 2 of Table 1 . We say that the 8 -cycles of this $H$-orbit are of type $I$ (see Figure 2).

Suppose now that $m=3$. With no loss of generality we can assume that the sequence 11100100 is obtained when traversing $C$ according to increasing subscripts of vertices and, in addition, that $c_{0} \in X_{0}$ and that the walk $\left(c_{0}, c_{1}, c_{2}, c_{3}\right)$ gives rise to the subsequence 010 . We first consider the possibility that $c_{1} \in X_{0}$. Then $c_{2}, c_{3} \in X_{1}$. We claim that this forces $c_{4} \in X_{1}$. Namely, if this is not the case, then $c_{4}, c_{5} \in X_{0}$, and so the fact that $c_{5} \rightarrow c_{6} \rightarrow c_{7} \leftarrow c_{0}$ implies that $C$ does not contain vertices from $X_{2}$, a contradiction. Therefore, $c_{4} \in X_{1}$ and hence $c_{5} \in X_{2}$. It is now easy to see that the only way for $C$ to have the required code is to have $c_{6} \in X_{2}$ and $c_{7} \in X_{0}$. Thus, $C$ is contained in the $H$-orbit of 8 -cycles whose representative is given in row 3 of 
Table 1. We say that the 8-cycles of this $H$-orbit are of type II (see Figure 2). Consider now the possibility that $c_{1} \notin X_{0}$, and so $c_{1} \in X_{2}$. It follows that $c_{2} \in X_{2}$ and $c_{3} \in X_{1}$. If $c_{4} \in X_{1}$, then $c_{5} \in X_{2}$, and then the only way for $C$ to have the required code is to have $c_{6} \in X_{2}$ and $c_{7} \in X_{0}$. Note that this 8-cycle is of type I. If however $c_{4} \in X_{0}$, then $c_{5} \in X_{0}$, and then the only way for $C$ to have the required code is to have $c_{6}, c_{7} \in X_{1}$. Thus $C$ is in the $H$-orbit of 8 -cycles from row 4 of Table 1 . We say that such 8-cycles are of type III (see Figure 2). To summarize, if $m=3$ we can have up to four different types of 8-cycles with code 11100100: the generic ones and 8 -cycles of types I, II and III.

CASE 4: $C$ meets four orbits, say, with no loss of generality, $X_{0}, X_{1}, X_{2}$ and $X_{3}$.

Observe first that no 8-cycle with code 11100100 exists if $m>4$. Namely, in this case an 8-cycle would necessarily have to contain precisely two vertices from each of the orbits $X_{0}, X_{1}, X_{2}$ and $X_{3}$. But then the code of $C$ would contain 1111 as a subsequence, which is impossible. We can therefore assume that $m=4$. Because of the particular code of $C$ it is clear that $C$ has at most three consecutive outer edges. Hence, either all the outer edges of $C$ give rise to digit 1 in the code of $C$ or they all give rise to digit 0 . It follows that $C$ has precisely four inner and four outer edges. If the outer edges give rise to digit 1 in the code 11100100 of $C$, then $C$ belongs to the $H$-orbit of 8-cycles from row 5 of Table 1 . We say that such 8-cycles are of type $I V$ (see Figure 2). If on the other hand the outer edges give rise to digit 0 in the code 11100100 of $C$, then $C$ belongs to the $H$-orbit of 8 -cycles from row 6 of Table 1 . We say that the 8 -cycles of this $H$-orbit are of type $V$ (see Figure 2 ).

CASE 5: $C$ meets more than four orbits.

It is easy to see that no such 8-cycle exists.

Proposition 5.3 Let $X$ be a connected quartic half-arc-transitive weak $(m, n)$-metacirculant of Class II and let $v \in V(X)$. Then (Aut $X)_{v} \cong \mathbb{Z}_{2}$ or possibly (Aut $\left.X\right)_{v} \cong$ $\mathbb{Z}_{2} \times \mathbb{Z}_{2}$ in which case $m=4$.

Proof: Let $\rho, \sigma \in \operatorname{Aut} X$ be such that $X$ is a weak $(m, n)$-metacirculant of Class II relative to the ordered pair $(\rho, \sigma)$ and that all the assumptions made in the third paragraph of this section hold. Moreover, adopt the notation introduced in this section and let $C$ denote the generic 8 -cycle from row 1 of Table 1 . We distinguish two cases depending on whether $m$ equals 4 or not.

Table 1 Possible $H$-orbits of 8-cycles of code 11100100.

\begin{tabular}{|c|c|c|c|}
\hline Row & Type & A representative & Condition \\
\hline 1 & generic & $u_{0}^{0} u_{0}^{s} u_{1}^{s} u_{1}^{s+r s} u_{0}^{s+r s} u_{0}^{r s} u_{1}^{r s} u_{1}^{0}$ & none \\
\hline 2 & type I & $u_{0}^{0} u_{1}^{0} u_{1}^{s r} u_{2}^{s r} u_{2}^{s\left(r-r^{2}\right)} u_{1}^{s\left(r-r^{2}\right)} u_{1}^{s\left(2 r-r^{2}\right)} u_{0}^{s\left(2 r-r^{2}\right)}$ & $s\left(1-2 r+r^{2}\right)=0$ \\
\hline 3 & type II & $u_{0}^{0} u_{1}^{0} u_{1}^{s r} u_{2}^{s r} u_{2}^{s\left(r-r^{2}\right)} u_{2}^{s\left(r-2 r^{2}\right)} u_{0}^{s\left(r-2 r^{2}\right)+t} u_{0}^{s\left(-1+r-2 r^{2}\right)+t}$ & $m=3$ and $t=s\left(2-r+r^{2}\right)$ \\
\hline 4 & type III & $u_{0}^{0} u_{0}^{s} u_{1}^{s} u_{1}^{s(1+r)} u_{0}^{s(1+r)} u_{2}^{s(1+r)-t} u_{2}^{s\left(1+r+r^{2}\right)-t} u_{1}^{s\left(1+r+r^{2}\right)-t}$ & $m=3$ and $t=s\left(1+r+r^{2}\right)$ \\
\hline 5 & type IV & $u_{0}^{0} u_{1}^{0} u_{2}^{0} u_{3}^{0} u_{3}^{-s r^{3}} u_{3}^{-2 s r^{3}} u_{0}^{-2 s r^{3}+t} u_{0}^{s\left(-1-2 r^{3}\right)+t}$ & $m=4$ and $t=s\left(2+2 r^{3}\right)$ \\
\hline 6 & type V & $u_{0}^{0} u_{0}^{s} u_{0}^{2 s} u_{0}^{3 s} u_{3}^{3 s-t} u_{2}^{3 s-t} u_{2}^{s\left(3+r^{2}\right)-t} u_{1}^{s\left(3+r^{2}\right)-t}$ & $m=4$ and $t=s\left(3+r^{2}\right)$ \\
\hline
\end{tabular}


Fig. 2 Possible types of 8-cycles having code 11100100.

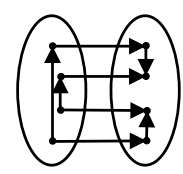

Generic
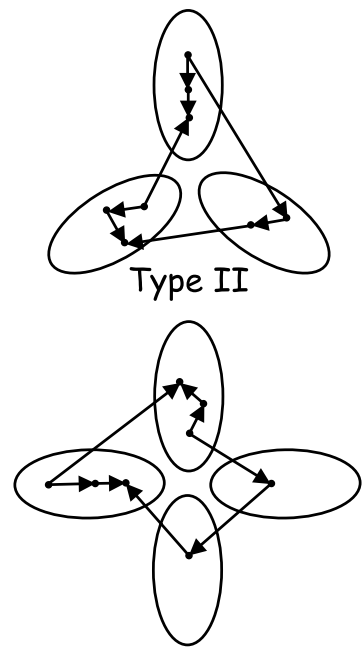

Type IV

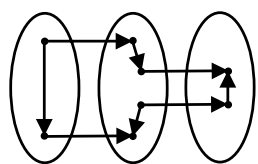

Type I
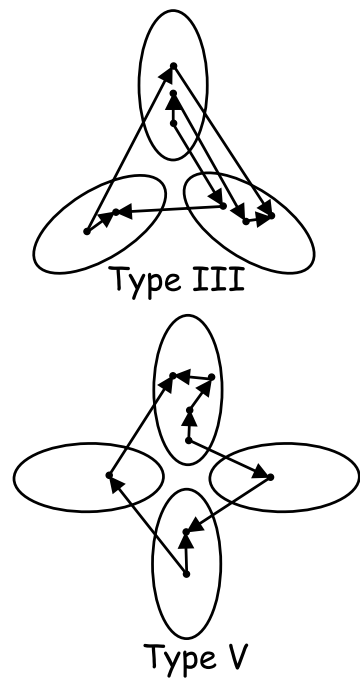

CASE $1: m \neq 4$.

If the stabilizer (Aut $X)_{u_{0}^{s}}$ is not isomorphic to $\mathbb{Z}_{2}$, then there exists an automorphism $\varphi$ of $X$ which fixes $u_{0}^{0}$ and $u_{0}^{s}$, and maps $u_{1}^{s}$ to $u_{0}^{2 s}$. Therefore, $C \varphi$ is an 8-cycle (with code 11100100) containing three consecutive vertices $\left(u_{0}^{0}, u_{0}^{s}\right.$ and $\left.u_{0}^{2 s}\right)$ in a single orbit of $\langle\rho\rangle$. It follows, by Lemma 5.2, that $m=3$ and that $C \varphi$ is of type II. Thus we must have $u_{1}^{s+r s} \varphi=u_{2}^{2 s-t}$. But this is impossible since then $u_{1}^{s} \rightarrow u_{1}^{s+r s}$ and $u_{1}^{s} \varphi \leftarrow u_{1}^{s+r s} \varphi$. It follows that (Aut $\left.X\right)_{v} \cong \mathbb{Z}_{2}$, as claimed.

\section{CASE 2: $m=4$.}

Suppose that $\left|(\operatorname{Aut} X)_{v}\right|>2$. Then there exists an automorphism $\varphi$ of $X$ fixing $u_{0}^{s+r s}$ and $u_{1}^{s+r s}$, and interchanging $u_{0}^{r s}$ and $u_{3}^{s+r s-t}$. This implies that $u_{1}^{r s} \varphi=$ $u_{3}^{s+r s+r^{3} s-t}, u_{1}^{0} \varphi=u_{2}^{s+r s+r^{3} s-t}$ and $u_{1}^{s} \varphi=u_{1}^{s}$. It follows that $C \varphi$ is of type V. Therefore, 8-cycles of type $\mathrm{V}$ exist in $X$.

To complete the proof we now show that the only automorphism of $X$ fixing a vertex and all of its neighbors is the identity. To this end let $\varphi \in \operatorname{Aut} X$ be an automorphism fixing $u_{0}^{0}$ and its four neighbors $u_{0}^{-s}, u_{0}^{s}, u_{3}^{-t}$ and $u_{1}^{0}$. There exists a unique 8cycle $C^{\prime}$ with code 11100100 containing vertices $u_{0}^{-s}, u_{0}^{0}, u_{0}^{s}$ and $u_{0}^{2 s}$. (It is of type V.) Since $\varphi$ fixes the first three of these four vertices, and since $u_{0}^{-s} \rightarrow u_{0}^{0} \rightarrow u_{0}^{s} \rightarrow u_{0}^{2 s} \varphi$ is a directed path of $C^{\prime} \varphi$, the 8-cycle $C^{\prime} \varphi$ is of type V. Consequently, $\varphi$ fixes all of its vertices pointwise. In particular $u_{0}^{2 s} \varphi=u_{0}^{2 s}$. It is now clear that $\varphi$ fixes $u_{0}^{s}$ and all of its neighbors. Continuing inductively, we see that $\varphi$ fixes every vertex of form $u_{0}^{j s}$ 
and all of its neighbors. Considering again the generic 8-cycle $C$. Since $\varphi$ fixes its vertices $u_{0}^{0}, u_{0}^{s}, u_{0}^{r s}, u_{0}^{s+r s}$ and all of their neighbors, it fixes $C$ pointwise. It follows that $\varphi$ fixes $u_{1}^{0}$ and all of its neighbors. Since $X$ is connected a repeated use of the above argument finally shows that $\varphi$ is the identity, as required. It is now clear, that $\mid(\text { Aut } X)_{v} \mid=4$, and so $(\text { Aut } X)_{v} \cong \mathbb{Z}_{2} \times \mathbb{Z}_{2}$, as claimed.

Remark: In fact, as we shall see in the proof of Theorem 5.1, the vertex stabilizer cannot be isomorphic to $\mathbb{Z}_{2} \times \mathbb{Z}_{2}$.

Proposition 5.4 Let $X$ be a connected quartic half-arc-transitive weak $(m, n)$-metacirculant of Class II. Then $m$ divides $n$ and moreover, there exist $r \in \mathbb{Z}_{n}^{*}$ and $t \in \mathbb{Z}_{n}$, satisfying (21) and (24), such that $X \cong \mathcal{Y}(m, n ; r, t)$.

Proof: Let $\rho, \sigma \in \operatorname{Aut} X$ be such that $X$ is a weak $(m, n)$-metacirculant of Class II relative to the ordered pair $(\rho, \sigma)$ and that all the assumptions made in the third paragraph of this section hold. Moreover, adopt the notation introduced in this section. Let $d_{s}$ denote the order of $s$ in $\mathbb{Z}_{n}$. There exist unique integers $a \geq 0$ and $b \in\{0,1, \ldots, m-1\}$ such that $d_{s}=a m+b$. Let $C_{0}$ denote the directed $d_{s^{-}}$ cycle $u_{0}^{0} u_{0}^{s} u_{0}^{2 s} \cdots u_{0}^{\left(d_{s}-1\right) s}$. By Proposition 5.3 there exists a unique automorphism $\tau \in \operatorname{Aut} X$, which fixes $u_{0}^{0}$, interchanges $u_{0}^{s}$ and $u_{1}^{0}$, and interchanges $u_{0}^{-s}$ and $u_{m-1}^{-t}$. We claim, that the image $C_{0} \tau$ of $C_{0}$ under $\tau$ is the directed $d_{s}$-cycle at $u_{0}^{0}$ consisting only of outer edges. Suppose this does not hold. Then there exists a smallest $k \in\left\{1,2, \ldots, d_{s}-1\right\}$ such that $\tau$ maps the inner edge $u_{0}^{k s} u_{0}^{(k+1) s}$ to an inner edge. Since $H=\langle\rho, \sigma\rangle$ acts transitively on $V(X)$, there exists an automorphism $\varphi \in H$ such that $u_{0}^{k s} \tau=u_{0}^{k s} \varphi$. The orbits $X_{i}$ of $\rho$ are blocks of imprimitivity for $H$, and so it is clear that $\varphi$ maps inner edges to inner edges. Therefore, we also have $u_{0}^{(k+1) s} \tau=u_{0}^{(k+1) s} \varphi$. However, as $\tau \notin H, \tau \varphi^{-1}$ is a nontrivial automorphism of $X$ fixing an edge. Hence, Proposition 5.3 implies that (Aut $X)_{u_{0}^{0}} \cong \mathbb{Z}_{2} \times \mathbb{Z}_{2}$ and that $m=4$. Moreover, following its proof we see that 8-cycles with code 11100100 of type $\mathrm{V}$ exist. In particular there exists a unique 8 -cycle $C_{1}$ of type $\mathrm{V}$ containing vertices $u_{0}^{-s}, u_{0}^{0}, u_{0}^{s}$ and $u_{0}^{2 s}$. Since $\tau$ maps the first three vertices to $u_{3}^{-t}, u_{0}^{0}$ and $u_{1}^{0}$, respectively, it is clear that $C_{1} \tau$ is of type IV. It follows that $u_{0}^{2 s} \tau=u_{2}^{0}$. We now repeatedly use this argument on 8-cycles of type $\mathrm{V}$ containing vertices $u_{0}^{(i-1) s}, u_{0}^{i s}, u_{0}^{(i+1) s}$ and $u_{0}^{(i+2) s}$ to finally prove that the edge $u_{0}^{k s} u_{0}^{(k+1) s}$ gets mapped to an outer edge, a contradiction which proves our claim.

Observe that the fact that $C_{0} \tau$ is the directed $d_{s}$-cycle at $u_{0}^{0}$ consisting only of outer edges implies that $u_{b}^{a t}=u_{0}^{0}$, and so $b=0$ and $a t=0$. In particular, this shows that the order of $t$ in $\mathbb{Z}_{n}$ divides $a$. Since $d_{s}=a m$ is the order of $s$ in $\mathbb{Z}_{n}$, we have that the order of $t$ in $\mathbb{Z}_{n}$ divides $d_{s}$. Therefore, $\langle t\rangle$ is a subgroup of $\langle s\rangle$. However, the connectedness of $X$ implies that $\langle s, t\rangle=\mathbb{Z}_{n}$, and so $\langle s\rangle=\mathbb{Z}_{n}$, that is $\operatorname{gcd}(n, s)=1$. It is now clear that $X \cong \mathcal{Y}(m, n ; r, t)$. Finally, the equation $n=d_{s}=a m$ implies that $m$ divides $n$. 
Remark: Note that Proposition 5.4 implies that one can assume $s=1$ in (19), that is, $u_{0}^{0} \rightarrow u_{0}^{1}$. For the rest of this section we therefore let $s=1$.

For future reference we record the nature of the action of the automorphism $\tau$ from the proof of the above proposition.

Lemma 5.5 With the notation introduced in this section let $d_{m}$ be the unique integer such that $n=m d_{m}$. Then for every $i \in \mathbb{Z}_{m}$ and every $j \in \mathbb{Z}_{n}$ there exist unique integers $a \in\left\{0,1, \ldots, d_{m}-1\right\}$ and $b \in\{0,1, \ldots, m-1\}$ such that $j=(a m+b) r^{i}$ in $\mathbb{Z}_{n}$. Moreover, the unique automorphism $\tau$ of $X$ fixing $u_{0}^{0}$, interchanging $u_{0}^{1}$ and $u_{1}^{0}$, and interchanging $u_{0}^{-1}$ and $u_{m-1}^{-t}$, maps according to the rule $u_{i}^{j} \tau=u_{b}^{i+a t}$.

PROOF: Observe first that since $r \in \mathbb{Z}_{n}^{*}$ the existence of unique $a$ and $b$ is clear. The proof of Proposition 5.4 shows that $\tau$ maps the inner edges of $X_{0}$ to outer edges. Therefore it maps the outer edges connecting $X_{0}$ to $X_{1}$ to inner edges. Continuing inductively we can see that $\tau$ interchanges inner edges with outer edges. It is now clear that $u_{i}^{0} \tau=u_{0}^{i}$ and that $u_{i}^{r^{i}} \tau=u_{1}^{i}, u_{i}^{2 r^{i}} \tau=u_{2}^{i}$, etc. Finally, $u_{i}^{(a m+b) r^{i}} \tau=u_{b}^{i+a t}$, which completes the proof.

The fact that the permutation $\tau$ from Lemma 5.5 is an automorphism of $X$ puts some further restrictions on parameters $m, n, r, t$ of $X \cong \mathcal{Y}(m, n ; r, t)$. Consider the generic 8-cycle $C$ from row 1 of Table 1. Lemma 5.5 implies that $\tau$ maps $C$ to the 8 -cycle from row 2 of Table 1, in particular $C \tau$ is of type I. Therefore, 8-cycles of type I exist in $X$, and so

$$
(r-1)^{2}=0 .
$$

Consequently,

$r^{m}=((r-1)+1)^{m}=(r-1)^{m}+m(r-1)^{m-1}+\cdots+m(r-1)+1=m(r-1)+1$,

and so (21) implies that

$$
m(r-1)=0 .
$$

Let $d_{m}$ be as in Lemma 5.5. Then $\tau$ maps the directed $n$-cycle $u_{0}^{0} u_{0}^{1} u_{0}^{2} \ldots u_{0}^{n-1}$ to $u_{0}^{0} u_{1}^{0} u_{2}^{0} \ldots u_{m-1}^{\left(d_{m}-1\right) t}$, and so $d_{m} t=0$. Moreover, $d_{m}$ is the smallest such positive integer. It is thus clear that

$$
|m|=|t| \text { in } \mathbb{Z}_{n} \text {, and so }\langle m\rangle=\langle t\rangle .
$$

We now show that

$$
|m|=|t|>2 .
$$

Consider the permutation $\psi$ of $V(X)$ defined by the rule: $u_{i}^{j} \psi=u_{i}^{-j}$ for $i \in \mathbb{Z}_{m}$, $j \in \mathbb{Z}_{n}$. It is easy to see that $\psi$ is an automorphism of $X$ if and only if $2 t=0$. But as 
$\psi \rho$ interchanges adjacent vertices $u_{0}^{0}$ and $u_{0}^{1}$, Proposition 3.1 implies that $\psi$ cannot be an automorphism of $X$, and so $2 t \neq 0$, as claimed. Note also that

$$
r^{2} \notin\{ \pm 1\}
$$

for otherwise $r^{m-i}= \pm r^{i}$ for all $i \in \mathbb{Z}_{m}$, and so the permutation $\varphi$ of $V(X)$ mapping according to the rule $u_{0}^{j} \varphi=u_{0}^{-j}$ and $u_{i}^{j} \varphi=u_{m-i}^{-j-t}$, where $i \in \mathbb{Z}_{m} \backslash\{0\}$ and $j \in \mathbb{Z}_{n}$, is an automorphism of $X$. But this is impossible, since then the automorphism $\varphi \rho$ inverts adjacent vertices $u_{0}^{0}$ and $u_{0}^{1}$, which contradicts Proposition 3.1.

Another immediate consequence of the existence of the automorphism $\tau$ from Lemma 5.5 is the following lemma.

Lemma 5.6 With the notation introduced in this section let $n=m d_{m}$, where $m$ and $d_{m}$ have the same role as in the statement of Lemma 5.5. Let $a \in\left\{0,1, \ldots, d_{m}-\right.$ $1\}$ and let $b \in\{0,1, \ldots, m-1\}$. Then there exists a unique $a^{\prime} \in\left\{0,1, \ldots, d_{m}-1\right\}$ such that $\left(a-a^{\prime}\right) m=\left(a^{\prime}-a\right) t=b(r-1)$. In particular, there exists a unique $a^{\prime} \in$ $\left\{0,1, \ldots, d_{m}-1\right\}$ such that $-a^{\prime} m=a^{\prime} t=r-1$, and so $m$ divides $r-1$, that is, $r-1 \in\langle m\rangle \leq \mathbb{Z}_{n}$.

PROOF: Lemma 5.5 implies that there exist unique $a^{\prime} \in\left\{0,1, \ldots, d_{m}-1\right\}$ and $b^{\prime} \in$ $\{0,1, \ldots, m-1\}$ such that $a m+b=\left(a^{\prime} m+b^{\prime}\right) r$ in $\mathbb{Z}_{n}$. Let $\tau$ be as in Lemma 5.5. Since $\tau$ maps the outer edge $u_{0}^{a m+b} u_{1}^{\left(a^{\prime} m+b^{\prime}\right) r}$ to the inner edge $u_{b}^{a t} u_{b^{\prime}}^{1+a^{\prime} t}$, we have that $b^{\prime}=b$ and that $1+a^{\prime} t=a t+r^{b}$. By (26) we have $r^{b}-1=b(r-1)$. Taking into account equations (24) and (27), we see that $\left(a-a^{\prime}\right) m=b(r-1)$ and $\left(a^{\prime}-a\right) t=$ $b(r-1)$, as required. Plugging in the values $b=1$ and $a=0$ we get that $-a^{\prime} m=$ $r-1=a^{\prime} t$ in $\mathbb{Z}_{n}$. Since $m$ divides $n$, this implies that $m$ divides $r-1$, completing the proof.

We are now ready to investigate possible attachment numbers of $X$. To this end let us inspect the two alternating cycles containing $u_{0}^{0}$. The directions of edges on the one on which $u_{0}^{0}$ is the tail of the two incident edges are

$$
u_{0}^{0} \rightarrow u_{1}^{0} \leftarrow u_{1}^{-r} \rightarrow u_{2}^{-r} \leftarrow \cdots \rightarrow u_{0}^{t-r-r^{2}-\cdots-r^{m-1}} \leftarrow u_{0}^{t-1-r-\cdots-r^{m-1}} \rightarrow \cdots
$$

The directions of edges on the other alternating cycle containing $u_{0}^{0}$ are

$$
u_{0}^{0} \leftarrow u_{0}^{-1} \rightarrow u_{1}^{-1} \leftarrow u_{1}^{-1-r} \rightarrow \cdots \leftarrow u_{m-1}^{-1-r-\cdots-r^{m-1}} \rightarrow u_{0}^{t-1-r-\cdots-r^{m-1}} \leftarrow \cdots
$$

It is therefore clear that $X$ is tightly attached if and only if there exists some $k \in \mathbb{Z}_{n}$ such that $-1=k\left(t-\left(1+r+r^{2}+\cdots+r^{m-1}\right)\right)-r$, that is, if and only if

$$
r-1=k\left(t-\left(1+r+r^{2}+\cdots+r^{m-1}\right)\right) \quad \text { for some } \quad k \in \mathbb{Z}_{n}
$$


Let us also note that (26) implies that

$$
\begin{aligned}
1+r+\cdots+r^{m-1} & =1+(r-1)+1+\cdots+((r-1)+1)^{m-1} \\
& =m+(1+2+\cdots+m-1)(r-1) \\
& =m+\frac{m(m-1)}{2}(r-1) .
\end{aligned}
$$

Lemma 5.7 With the notation introduced in this section let $n=m d_{m}$, where $m$ and $d_{m}$ have the same role as in the statement of Lemma 5.5. If either $m$ or $d_{m}$ is odd then $X$ is tightly attached.

PROOF: We claim that in each of these two cases $1+r+\cdots+r^{m-1}=m$ and $\langle 2(r-$ $1)\rangle=\langle r-1\rangle$. Suppose first that $m$ is odd. Then (27) and (32) imply that $1+r+\cdots+$ $r^{m-1}=m$. Moreover, (27) and the fact that $m$ is odd imply that $\langle 2(r-1)\rangle=\langle r-1\rangle$. Suppose now that $d_{m}$ is odd but $m$ is even. Then Lemma 5.6 implies that $\langle 2(r-1)\rangle=$ $\langle r-1\rangle$. Therefore, $\frac{m}{2}(r-1)=0$, and so $1+r+\cdots+r^{m-1}=m$, which proves our claim.

By Lemma 5.6 there exists a unique integer $a^{\prime} \in\left\{0,1, \ldots, d_{m}-1\right\}$ such that $-a^{\prime} m=a^{\prime} t=r-1$, and so $a^{\prime}(t-m)=2(r-1)$. Combining together the above claim and (31), we see that $X$ is tightly attached.

Lemma 5.8 With the notation introduced in this section let $n=m d_{m}$, where $m$ and $d_{m}$ have the same role as in the statement of Lemma 5.5. Further, let $n=2^{i} n_{1}$ where $n_{1}$ is odd. If $i \leq 2$ then $X$ is tightly attached.

Proof: By Lemma 5.7 we can assume that both $m$ and $d_{m}$ are even. Hence $i=2$ and $m \equiv 2(\bmod 4)$. As $m(r-1)=0$, we either have $\frac{m}{2}(r-1)=0$ or $\frac{m}{2}(r-1)=\frac{n}{2}$. We consider these two cases separately.

CASE 1: $\frac{m}{2}(r-1)=0$.

Then (32) implies that $1+r+\cdots+r^{m-1}=m$. Moreover, as $\frac{m}{2}$ is odd we have $\langle 2(r-1)\rangle=\langle r-1\rangle$. By Lemma 5.6 there exists a unique $a^{\prime} \in\left\{0,1, \ldots, d_{m}-1\right\}$ such that $-a^{\prime} m=a^{\prime} t=r-1$, and so, as in the proof of Lemma 5.7, $X$ is tightly attached by (31).

CASE 2: $\frac{m}{2}(r-1)=\frac{n}{2}$.

Since $n$ is even, $r$ is odd, and so $r-1$ is even. Moreover, as $\frac{m}{2}(r-1)=\frac{n}{2}$, we have that $r-1 \equiv 2(\bmod 4)$. Let $c=t-m+\frac{n}{2}$, that is, $c=t-\left(1+r+\cdots+r^{m-1}\right)$ in view of (32). By (31), $X$ is tightly attached if and only if $r-1 \in\langle c\rangle$. By Lemma 5.6 there exists a unique $a^{\prime} \in\left\{0,1, \ldots, d_{m}-1\right\}$ such that $-a^{\prime} m=a^{\prime} t=r-1$. Note that $a^{\prime}$ is odd, and so $a^{\prime} \frac{n}{2}=\frac{n}{2}$. Consequently $a^{\prime} c=2(r-1)+\frac{n}{2}$. To show that $X$ is tightly attached it thus suffices to see that $\left\langle 2(r-1)+\frac{n}{2}\right\rangle=\langle r-1\rangle$. It is clear that $2(r-1)+\frac{n}{2} \equiv 2(\bmod 4)$. Moreover, $\frac{n}{2} \in\langle r-1\rangle$, and so $2(r-1)+\frac{n}{2} \in\langle r-1\rangle$. Finally, let $p^{t}$ be any odd prime power dividing $2(r-1)+\frac{n}{2}$ and $n$. Since $p$ is odd, $p^{t}$ divides $\frac{n}{2}$, and so it also divides $r-1$. Therefore, a prime power $p^{t}$ dividing $n$ divides $r-1$ if and only if it divides $2(r-1)+\frac{n}{2}$. Since $\mathbb{Z}_{n}$ is a cyclic group, we indeed have $\left\langle 2(r-1)+\frac{n}{2}\right\rangle=\langle r-1\rangle$, as required. 
Combining together the results of this section, the proof of Theorem 5.1 is now at hand.

\section{PROOF OF THEOREM 5.1:}

Throughout this proof we adopt the notation of this section. Except for the existence of a unique $c \in\left\{0,1, \ldots, d_{m}-1\right\}$ for which $t=\mathrm{cm}$ and $m=c t$, claims (i), (iii) and (iv) follow from (20), (21), (24), Proposition 5.4, Lemma 5.6 and (29). To show that a unique such $c$ exists, observe that there exists a unique $c \in\left\{0,1, \ldots, d_{m}-1\right\}$ for which $t=\mathrm{cm}$. Plugging in the values $i=0$ and $j=t$ in Lemma 5.5 we get that $u_{0}^{t} \tau=u_{0}^{c m} \tau=u_{0}^{c t}$. On the other hand, letting $i=0$ and $j=m$, we get that $u_{0}^{m} \tau=u_{0}^{t}$, and so $u_{0}^{t} \tau=u_{0}^{m} \tau^{2}=u_{0}^{m}$, which gives $m=c t$, as required.

Let us now prove (ii). By contradiction, assume that (Aut $X)_{v}$ is not isomorphic to $\mathbb{Z}_{2}$. Then by Proposition 5.3, (Aut $\left.X\right)_{v} \cong \mathbb{Z}_{2} \times \mathbb{Z}_{2}$ and $m=4$. Moreover, the proof of Proposition 5.3 reveals that 8-cycles of type $\mathrm{V}$ exist (see Table 1). In particular $3+r^{2}=t$, and so equation (26) implies that $2+2 r=t$. Note also that (26) and (30) combined together imply that $2(r-1) \neq 0$. By (27) we thus have

$$
2(r-1)=\frac{n}{2} \quad \text { and } \quad t=\frac{n}{2}+4 .
$$

Furthermore, Lemma 5.6 and (27) combined together imply that $n=16 n_{1}$ for some integer $n_{1}$. Thus either $r-1=\frac{n}{4}=4 n_{1}$ or $r-1=\frac{3 n}{4}=12 n_{1}$. Let $a^{\prime}$ be the unique element of $\left\{0,1, \ldots, 4 n_{1}-1\right\}$ such that $-a^{\prime} m=a^{\prime} t=r-1$, which exists by Lemma 5.6. As $-a^{\prime} m=r-1$, we either have $a^{\prime}=3 n_{1}$ or $a^{\prime}=n_{1}$. But then $a^{\prime} t$ equals either to $3 n_{1}\left(8 n_{1}+4\right)=12 n_{1}+8 n_{1}^{2}$ or to $n_{1}\left(8 n_{1}+4\right)=4 n_{1}+8 n_{1}^{2}$, respectively. Thus, in view of the equality $r-1=a^{\prime} t$, we have $8 n_{1}^{2}=8 n_{1}=\frac{n}{2}$ in either case, and so $n_{1}$ is odd, that is $n \equiv 16(\bmod 32)$. Note that this also forces $r \equiv 5(\bmod 8)$.

We now introduce a certain mapping $\varphi: V(X) \rightarrow V(X)$, which will be shown below to be an automorphism of $X$. The nature of the action of $\varphi$ will contradict half-arc-transitivity of $X$, which thus proves that $(\text { Aut } X)_{v} \cong \mathbb{Z}_{2}$, as claimed. Note that (26) and (33) imply that $r^{2}=\frac{n}{2}+1$, and so $1+r+r^{2}+r^{3}+t=1+r+\frac{n}{2}+$ $1+\frac{n}{2}+r+t=2 t=8$. Let $j \in \mathbb{Z}_{n}$. Then there exist unique $a \in\left\{0,1, \ldots, 2 n_{1}-1\right\}$ and $b \in\{0,1, \ldots, 7\}$ such that $j=8 a+b$. The action of $\varphi$ on $u_{i}^{j}, i \in \mathbb{Z}_{m}, j \in \mathbb{Z}_{n}$, is given in Table 2 and it depends on $i$ and $b$.

To see that $\varphi$ is in fact a permutation of $V(X)$, we only need to observe that it is injective. Consider the vertices of Table 2, which are of the form $u_{0}^{j}$. There are precisely eight such vertices. It can be seen that the congruencies modulo 8 of their superscripts are precisely the eight possibilities $0,1, \ldots, 7$. For instance, for $u_{0}^{-8 a}$ we have 0 , for $u_{0}^{-1-r-r^{3}-t-8 a}$ we have 1 , etc. Similarly, one can check that there are precisely eight vertices of the form $u_{i}^{j}$ in Table 2 for each $i=1,2,3$, and that the corresponding congruencies modulo 8 of their superscripts are again the eight possibilities $0,1, \ldots, 7$. This shows that $\varphi$ is indeed injective and thus also bijective. It remains to be seen that $\varphi$ preserves adjacency in $X$. For the outer edges connecting $X_{i}$ to $X_{i+1}$, where $i \neq 3$, and for the inner edges of $X_{0}$, this is clear, as one only needs to check that two consecutive vertices in a row or in column 0 , respectively, of Table 2 are adjacent. As for the other edges, using the facts that $t=\frac{n}{2}+4$, that $r^{2}=\frac{n}{2}+1$, 
Table 2 The entry in $b$-th row and $i$-th column represents the image $u_{i}^{j} \varphi$ in the case when $j=8 a+b$, where $a \in\left\{0,1, \ldots, 2 n_{1}-1\right\}$ and $b \in\{0,1, \ldots, 7\}$.

\begin{tabular}{l|l|l|l|l}
$b \backslash i$ & \multicolumn{1}{|c|}{0} & \multicolumn{1}{|c}{1} & \multicolumn{1}{|c}{2} & \multicolumn{1}{|c}{3} \\
\hline 0 & $u_{0}^{-8 a}$ & $u_{3}^{-t-8 a}$ & $u_{3}^{-r^{3}-t-8 a}$ & $u_{2}^{-r^{3}-t-8 a}$ \\
1 & $u_{0}^{-1-8 a}$ & $u_{0}^{-2-8 a}$ & $u_{3}^{-2-t-8 a}$ & $u_{3}^{-2-r^{3}-t-8 a}$ \\
2 & $u_{3}^{-1-t-8 a}$ & $u_{2}^{-1-t-8 a}$ & $u_{2}^{-1-r^{2}-t-8 a}$ & $u_{1}^{-1-r^{2}-t-8 a}$ \\
3 & $u_{3}^{-1-r^{3}-t-8 a}$ & $u_{3}^{-1-2 r^{3}-t-8 a}$ & $u_{2}^{-1-2 r^{3}-t-8 a}$ & $u_{2}^{-1-r^{2}-2 r^{3}-t-8 a}$ \\
4 & $u_{2}^{-1-r^{3}-t-8 a}$ & $u_{1}^{-1-r^{3}-t-8 a}$ & $u_{1}^{-1-r-r^{3}-t-8 a}$ & $u_{0}^{-1-r-r^{3}-t-8 a}$ \\
5 & $u_{2}^{-1-r^{2}-r^{3}-t-8 a}$ & $u_{2}^{-1-2 r^{2}-r^{3}-t-8 a}$ & $u_{1}^{-1-2 r^{2}-r^{3}-t-8 a}$ & $u_{1}^{-r^{2}-8(a+1)}$ \\
6 & $u_{1}^{-1-r^{2}-r^{3}-t-8 a}$ & $u_{0}^{-1-r^{2}-r^{3}-t-8 a}$ & $u_{0}^{-2-r^{2}-r^{3}-t-8 a}$ & $u_{3}^{-2-r^{2}-r^{3}-2 t-8 a}$ \\
7 & $u_{1}^{-8(a+1)}$ & $u_{1}^{-r-8(a+1)}$ & $u_{0}^{-r-8(a+1)}$ & $u_{0}^{-1-r-8(a+1)}$
\end{tabular}

and that $r \equiv 5 \quad(\bmod 8)$, checking that they are indeed mapped to edges of $X$ is just a matter of tedious computation. We leave the details to the reader. Therefore $\varphi$ is an automorphism of $X$. Since it fixes $u_{0}^{0}$ and maps $u_{0}^{1}$ to $u_{0}^{-1}$, it follows that $\varphi \rho$ interchanges adjacent vertices $u_{0}^{0}$ and $u_{0}^{1}$ of $X$, which contradicts Proposition 3.1. Thus $(\text { Aut } X)_{v} \cong \mathbb{Z}_{2}$ for all $v \in V(X)$, as claimed.

Finally, we prove (v). Let us suppose that $X$ is not tightly attached. By Lemma 5.7 $m$ and $d_{m}$ are both even and by Lemma 5.8, there exists some positive integer $n_{1}$ such that $n=8 n_{1}$. We show that $n_{1}>2$. Note first that part (iii) of this theorem, Proposition 3.2 and (29) combined together imply that $n_{1}>1$. Moreover, if $n_{1}=2$, then $m=4$, and so combining together (27) and (30) we have that $r \in\{5,13\}$. Therefore, (28) and Lemma 5.6 combined together imply that $t=12=\frac{n}{2}+4$. But then the mapping $\varphi$ introduced in the proof of part (ii) is an automorphism of $X$, a contradiction. Thus $n_{1}>2$, as claimed. Suppose now, that $n_{1}>2$ is odd and squarefree. Then (26) implies that $r-1=\frac{n}{2}$ (recall that $r \neq 1$ ). It follows that $r^{2}=((r-1)+1)^{2}=(r-1)^{2}+2(r-1)+1=1$, which contradicts $(30)$. This completes the proof of Theorem 5.1 .

There do exist connected quartic half-arc-transitive weak metacirculants of Class II which are not tightly attached. Using Theorem 5.1, a computer search has been performed revealing that there are precisely 18 such graphs of order not exceeding 1000. The smallest such graph is isomorphic to $\mathcal{Y}(4,48 ; 13,44)$, and is of order 192, has radius 12 and attachment number 3 . Table 3 contains some information 
about these 18 graphs. Observe that in view of existence of these graphs, Theorem 5.1 is best possible.

Table 3 All connected quartic half-arc-transitive weak metacirculants of Class II of order up to 1000 which are not tightly attached.

\begin{tabular}{||l|l|c|c||}
\hline order & graph & radius & att. num. \\
\hline 192 & $\mathcal{Y}(4,48 ; 13,44)$ & 12 & 3 \\
256 & $\mathcal{Y}(8,32 ; 9,24)$ & 16 & 8 \\
320 & $\mathcal{Y}(4,80 ; 21,76)$ & 20 & 5 \\
432 & $\mathcal{Y}(6,72 ; 13,66)$ & 18 & 9 \\
448 & $\mathcal{Y}(4,112 ; 29,108)$ & 28 & 7 \\
512 & $\mathcal{Y}(8,64 ; 9,56)$ & 32 & 16 \\
512 & $\mathcal{Y}(8,64 ; 25,56)$ & 32 & 16 \\
576 & $\mathcal{Y}(12,48 ; 13,36)$ & 12 & 3 \\
576 & $\mathcal{Y}(4,144 ; 37,140)$ & 36 & 9 \\
704 & $\mathcal{Y}(4,176 ; 45,172)$ & 44 & 11 \\
768 & $\mathcal{Y}(8,96 ; 25,56)$ & 16 & 8 \\
768 & $\mathcal{Y}(8,96 ; 25,88)$ & 48 & 24 \\
832 & $\mathcal{Y}(4,208 ; 53,204)$ & 52 & 13 \\
864 & $\mathcal{Y}(12,72 ; 13,60)$ & 36 & 18 \\
864 & $\mathcal{Y}(6,144 ; 25,30)$ & 18 & 9 \\
960 & $\mathcal{Y}(4,240 ; 61,44)$ & 12 & 3 \\
960 & $\mathcal{Y}(4,240 ; 61,76)$ & 20 & 5 \\
960 & $\mathcal{Y}(4,240 ; 61,236)$ & 60 & 15 \\
\hline
\end{tabular}

Recall that a weak $(m, n)$-metacirculant is not necessarily an $(m, n)$-metacirculant. However, as the next proposition shows, quartic half-arc-transitive weak metacirculants of Class II which are not tightly attached do have this property.

Proposition 5.9 Let $X$ be a connected quartic half-arc-transitive weak $(m, n)$-metacirculant of Class II which is not tightly attached. Then X is an $(m, n)$-metacirculant.

ProOF: Let $r, t \in \mathbb{Z}_{n}$ satisfy part (iv) of Theorem 5.1 , in particular $X \cong \mathcal{Y}(m, n ; r, t)$, and let the corresponding automorphisms be $\rho$ and $\sigma$. By part (v) of Theorem 5.1 we have that $m$ and $d_{m}$, where $n=m d_{m}$, are both even and that $n=8 n_{1}$, where $n_{1}>2$ is even or not squarefree. Observe that $X$ is a weak $(m, n)$-metacirculant relative to the ordered pair $\left(\rho, \sigma \rho^{k}\right)$ for any $k \in \mathbb{Z}_{n}$. We show that there exists some $k \in \mathbb{Z}_{n}$ for which $\sigma \rho^{k}$ is of order $m$, which then completes the proof.

Let $k \in \mathbb{Z}_{n}$. Since $\sigma^{m}=\rho^{t}$, equation (1) implies that

$$
\left(\sigma \rho^{k}\right)^{m}=\sigma^{m} \rho^{k\left(1+r+\cdots+r^{m-1}\right)}=\rho^{t+k\left(1+r+\cdots+r^{m-1}\right)} .
$$

Combining together (27) and (32), we have two possibilities for $1+r+\cdots+r^{m-1}$. If $1+r+\cdots+r^{m-1}=m$, then an appropriate $k$ exists by (28). We can thus assume that $1+r+\cdots+r^{m-1}=m+\frac{n}{2}$, that is, $\frac{m}{2}(r-1)=\frac{n}{2}$. Since $d_{m}$ is even, it is clear that $\left\langle m+\frac{n}{2}\right\rangle \leq\langle m\rangle$. If the order $d_{0}$ of $m+\frac{n}{2}$ in $\mathbb{Z}_{n}$ is also even, then $d_{0}=d_{m}$. Thus 
$\left\langle m+\frac{n}{2}\right\rangle=\langle m\rangle$, and we are done in view of (28). Suppose then that $d_{0}$ is odd, that is $d_{m}=2 d_{0}$. Since $m \frac{r-1}{2}=\frac{n}{2}$, we have that $r-1 \equiv 2(\bmod 4)$. But then $(r-1)^{2} \equiv$ $4(\bmod 8)$ which contradicts $(26)$ and the fact that $n \equiv 0(\bmod 8)$. This shows that an appropriate $k \in \mathbb{Z}_{n}$ does exist, as claimed.

To wrap up this section we construct an infinite family of connected quartic halfarc-transitive weak metacirculants of Class II which are not tightly attached. These graphs are constructed as regular $\mathbb{Z}_{p}$-covers, $p$ a prime, of the graph $\mathcal{Y}(4,48 ; 13,44)$, the smallest example of such graphs.

Construction 5.10 Let $X$ denote the graph $\mathcal{Y}(4,48 ; 13,44)$ and let $D_{X}$ denote the oriented graph corresponding to the half-arc-transitive action of Aut $X$ on $X$ in which $u_{0}^{0} \rightarrow u_{0}^{1}$. Let $p \geq 5$ be a prime. Following the theory developed in [19] we construct a regular $\mathbb{Z}_{p}$-cover of $X$ by voltage assignments from the cyclic group $\mathbb{Z}_{p}$, letting the voltage of each dart corresponding to an oriented edge of $D_{X}$ be 1 . Let $\mathcal{C}_{p}(X)$ denote the obtained $\mathbb{Z}_{p}$-cover. Note that since $X$ is half-arc-transitive, any automorphism of $X$ maps a cycle of $X$ with trivial net voltage to a cycle of $X$ with trivial net voltage. By [19, Corollary 7.2], the automorphism group Aut $X$ lifts, that is, there exists a group $\tilde{A} \leq \operatorname{Aut}_{p}(X)$ projecting to Aut $X$ and acting half-arc-transitively on $\mathcal{C}_{p}(X)$. We now show that $\mathcal{C}_{p}(X)$ is a connected quartic half-arc-transitive weak metacirculant of Class II with radius 12 and attachment number 3 . This will establish the existence of infinitely many connected quartic half-arc-transitive weak metacirculants of Class II which are not tightly attached.

Denote the vertices of $\mathcal{C}_{p}(X)$ by $\left\{{ }^{k} u_{i}^{j} \mid i \in \mathbb{Z}_{4}, j \in \mathbb{Z}_{48}, k \in \mathbb{Z}_{p}\right\}$. Let $D_{\mathcal{C}_{p}(X)}$ be the oriented graph corresponding to the half-arc-transitive action of $\tilde{A}$ on $\mathcal{C}_{p}(X)$ such that ${ }^{0} u_{0}^{0} \rightarrow{ }^{1} u_{0}^{1}$. The orientations of the edges of $D_{\mathcal{C}_{p}(X)}$ are thus

$$
{ }^{k} u_{i}^{j} \rightarrow\left\{\begin{array}{c}
{ }^{k+1} u_{i+1}^{j} ; i \neq 3 \\
{ }^{k+1} u_{0}^{j+44} ; i=3
\end{array} \quad \text { and } \quad{ }^{k} u_{i}^{j} \rightarrow{ }^{k+1} u_{i}^{j+13^{i}}\right.
$$

where $i \in \mathbb{Z}_{4}, j \in \mathbb{Z}_{48}$ and $k \in \mathbb{Z}_{p}$. We proceed by proving a series of claims.

CLAIM 1: There exist $r \in \mathbb{Z}_{48 p}^{*}$ and $t \in \mathbb{Z}_{48 p}$ such that $\mathcal{C}_{p}(X) \cong \mathcal{Y}(4,48 p ; r, t)$. For any $l \in\{0,1, \ldots, 48 p-1\}$ let $\alpha(l)=(j, k)$, where $j \in\{0,1, \ldots, 47\}$ and $k \in\{0,1, \ldots, p-1\}$ are such that $l \equiv j(\bmod 48)$ and $l \equiv k(\bmod p)$. Note that $\alpha$ is a well defined mapping. Moreover, since $p \geq 5$ is a prime we have $\operatorname{GCD}(48, p)=1$, and so $\alpha$ gives a $1-1$ correspondence of $\{0,1, \ldots, 48 p-1\}$ and $\{0,1, \ldots, 47\} \times$ $\{0,1, \ldots, p-1\}$, and so it also gives a $1-1$ correspondence of $\mathbb{Z}_{48 p}$ and $\mathbb{Z}_{48} \times \mathbb{Z}_{p}$. In fact, $\alpha$ is an isomorphism of these Abelian groups. Let $r \in \mathbb{Z}_{48 p}$ be the unique element given by this bijective correspondence such that $\alpha(r)=(13,1)$. Note that then $r \in \mathbb{Z}_{48 p}^{*}$. Similarly let $t \in \mathbb{Z}_{48 p}$ be the unique element such that $\alpha(t)=(44,4)$. We now show that $\mathcal{C}_{p}(X) \cong \mathcal{Y}(4,48 p ; r, t)$. Let the vertex set of $\mathcal{Y}(4,48 p ; r, t)$ be $\left\{v_{i}^{j} \mid i \in \mathbb{Z}_{4}, j \in \mathbb{Z}_{48 p}\right\}$, with edges as in Example 2.3. We let $\varphi: \mathcal{C}_{p}(X) \rightarrow$ $\mathcal{Y}(4,48 p ; r, t)$ be the mapping defined by the rule $\varphi:{ }^{k} u_{i}^{j} \mapsto v_{i}^{l}$, where $l \in \mathbb{Z}_{48 p}$ is 
the unique element such that $\alpha(l)=(j, k-i)$. Since $\alpha$ is a bijection $\varphi$ is a bijection as well. We leave the easy verification that $\varphi$ is an isomorphism of graphs to the reader.

Claim 2: $\operatorname{Aut}_{p}(X)=\tilde{A}$.

By Claim 1 we have that $\mathcal{C}_{p}(X) \cong \mathcal{Y}(4,48 p ; r, t)$. It may be checked that $X$ has girth 8. As $\mathcal{C}_{p}(X)$ is a regular $\mathbb{Z}_{p}$-cover of $X$ and since generic 8-cycles of $X$ have trivial net voltage, it is clear that $\mathcal{C}_{p}(X)$ also has girth 8 . Moreover, any 8-cycle of $\mathcal{C}_{p}(X)$ projects to an 8 -cycle of $X$ via the corresponding covering projection. It thus follows that the only 8 -cycles of $\mathcal{C}_{p}(X)$ are the lifts of 8-cycles of $X$ with net voltage 0 . We now investigate all such 8-cycles of $X$.

It turns out that $X$ has precisely four Aut $X$-orbits of 8 -cycles with net voltage 0 . These are the Aut $X$-orbit $\mathcal{H}_{1}$, which contains generic 8-cycles and 8-cycles of type I (see Table 1), the Aut $X$-orbit $\mathcal{H}_{2}$ containing the 8-cycle $u_{0}^{0} u_{0}^{1} u_{0}^{2} u_{3}^{2-t} u_{2}^{2-t} u_{2}^{2-r^{2}-t}$ $u_{2}^{2-2 r^{2}-t} u_{3}^{2-2 r^{2}-t}$, the Aut $X$-orbit $\mathcal{H}_{3}$ containing the 8-cycle $u_{0}^{0} u_{0}^{1} u_{1}^{1} u_{2}^{1} u_{2}^{1+r^{2}} u_{1}^{1+r^{2}}$ $u_{1}^{1-r+r^{2}} u_{1}^{1-2 r+r^{2}}$ and the Aut $X$-orbit $\mathcal{H}_{4}$ containing the 8-cycle $u_{0}^{0} u_{0}^{1} u_{1}^{1} u_{1}^{1-r} u_{1}^{1-2 r}$ $u_{2}^{1-2 r} u_{2}^{1-2 r+r^{2}} u_{1}^{0}$. As the covering projection $p: \mathcal{C}_{p}(X) \rightarrow X$ gives a 1-1 correspondence between the 8-cycles of $\mathcal{C}_{p}(X)$ and the 8-cycles of $X$ with voltage 0 , we see that $\mathcal{C}_{p}(X)$ has four $\tilde{A}$-orbits of 8 -cycles. They are the lifts of the Aut $X$-orbits $\mathcal{H}_{i}$, and so we denote the $\tilde{A}$-orbits of 8-cycles of $\mathcal{C}_{p}(X)$ with $\tilde{\mathcal{H}}_{i}$ where $\mathcal{H}_{i}=p\left(\tilde{\mathcal{H}}_{i}\right)$.

Observe that $\mathcal{C}_{p}(X)$ has precisely four $\tilde{A}$-orbits of 2-paths (where no distinction is made on the orientation of these paths). Let $\mathcal{P}_{1}$ be the orbit containing the 2-path ${ }^{0} u_{0}^{01} u_{0}^{12} u_{0}^{2}$, let $\mathcal{P}_{2}$ be the orbit containing the 2-path ${ }^{0} u_{0}^{01} u_{0}^{12} u_{1}^{1}$, let $\mathcal{P}_{3}$ be the orbit containing the 2-path ${ }^{0} u_{0}^{0}{ }^{1} u_{0}^{10} u_{3}^{5}$ and let $\mathcal{P}_{4}$ be the orbit containing the 2-path ${ }^{1} u_{0}^{1}{ }^{0} u_{0}^{0}{ }^{1} u_{1}^{0}$. We now consider the bipartite graph $B i p_{2,8}$ whose vertex set is the union of the set of 8-cycles of $\mathcal{C}_{p}(X)$ and the set of 2-paths of $\mathcal{C}_{p}(X)$ with a 2-path $P$ being adjacent to an 8-cycle $C$ if and only if $C$ contains $P$. It is straightforward to check that the valency in $B i p_{2,8}$ of any 2-path from any one of $\mathcal{P}_{2}, \mathcal{P}_{3}$ and $\mathcal{P}_{4}$ is 8 , whereas the valency of any 2-path from $\mathcal{P}_{1}$ is 4 .

Suppose now that $\mathcal{C}_{p}(X)$ is arc-transitive. Following [22], we assign a letter $D$, $A^{+}$or $A^{-}$to an internal vertex $v$ of a 2-path $P$ in $\mathcal{C}_{p}(X)$ depending on whether $v$ is the head of one and tail of the other, head of both, or tail of both of the two incident edges in $P$, respectively. In such a way a code of length 2 can be assigned to each 3-path in $\mathcal{C}_{p}(X)$. For example, a directed 3-path is assigned the code $D^{2}$. Now, by [22, Lemma 2.1] either every automorphism of $\mathcal{C}_{p}(X)$ preserves or reverses the orientation of every edge or any two codes of length 2 are permutable (by an automorphism of $\left.\mathcal{C}_{p}(X)\right)$. If the latter occurs then there exists an automorphism of $\mathcal{C}_{p}(X)$ mapping some 3-path with code $D A^{+}$to some 3-path with code $A^{-} A^{+}$. It can be seen that any 3-path with code $D A^{+}$lies on some 8 -cycle from $\tilde{\mathcal{H}}_{4}$, whereas the only 8-cycles of $\mathcal{C}_{p}(X)$ which contain 3-paths with code $A^{-} A^{+}$are the 8-cycles from $\tilde{\mathcal{H}}_{1}$. Therefore, some automorphism of $\mathcal{C}_{p}(X)$, mapping an 8-cycle from $\tilde{\mathcal{H}}_{4}$ to an 8 -cycle from $\tilde{\mathcal{H}}_{1}$ exists. However, it may be verified that 8 -cycles from $\tilde{\mathcal{H}}_{4}$ contain two 2-paths from $\mathcal{P}_{1}$, whereas 8 -cycles from $\tilde{\mathcal{H}}_{1}$ contain none, which contradicts the fact that the 2-paths from $\mathcal{P}_{1}$ are the only 2-paths of $\mathcal{C}_{p}(X)$ of valency 4 in $B_{i p} p_{2,8}$. Therefore, every automorphism of $\mathcal{C}_{p}(X)$ either preserves the orientation of every edge of $\mathcal{C}_{p}(X)$ or reverses the orientation of every edge of $\mathcal{C}_{p}(X)$. 
More precisely, by assumption about arc-transitivity of $\mathcal{C}_{p}(X)$, there must exist an automorphism reversing the edge orientations. Let $\tilde{G}$ be the subgroup of index 2 of $\operatorname{Aut}_{p}(X)$ consisting of all the automorphisms preserving the orientation of the edges. If $\tilde{A}=\tilde{G}$, then $\tilde{A}$ is an index 2 subgroup of $\operatorname{Aut}_{p}(X)$ and is thus normal. Since $\mid$ Aut $X \mid=2 \cdot 4 \cdot 48=2^{7} \cdot 3$, we have that $|\tilde{A}|=p \cdot 2^{7} \cdot 3$, and so the group of covering transformations $C T_{X}=\mathbb{Z}_{p}$ is the unique $p$-Sylow subgroup of $\tilde{A}$. It is now clear that $C T_{X}$ is normal in $\operatorname{Aut}_{p}(X)$, and so $\operatorname{Aut}_{p}(X)$ projects. Thus $X$ is arc-transitive, a contradiction. Therefore $\left|\tilde{G}_{0} u_{0}^{0}\right| \geq 4$. However, this is also impossible as then $\mathcal{P}_{1}$ and $\mathcal{P}_{2}$ are contained in the same $\tilde{G}$-orbit which is clearly impossible in view of the valencies of the respective 2-paths in Bip $_{2,8}$. This contradiction finally proves that $\operatorname{Aut}_{p}(X)=\tilde{A}$, and so $\mathcal{C}_{p}(X)$ is half-arc-transitive, as claimed.

Claim 3: $Y_{p}$ has radius 12 and attachment number 3.

By Claim 2 the orientation of the edges of $\mathcal{C}_{p}(X)$ given by $\tilde{A}$ is in fact an orientation given by the half-arc-transitive action of $\operatorname{Aut}_{p}(X)$, and so it is clear that an alternating cycle of $X$ is lifted into an alternating cycle of $\mathcal{C}_{p}(X)$. Therefore, the radius of $\mathcal{C}_{p}(X)$ is 12 . The fact that the attachment number is 3 is now also clear.

Combining together Claims 1,2 and 3 we thus see that $\mathcal{C}_{p}(X)$ is a connected quartic half-arc-transitive weak metacirculant of Class II with radius 12 and attachment number 3 , as claimed. Note that the graph $\mathcal{Y}(4,240 ; 61,44)$ from Table 3 is isomorphic to $\mathcal{C}_{5}(X)$.

\section{Conclusions}

Half-arc-transitive metacirculants of Classes III and IV will be studied in a sequel to this paper, together with their connection to graphs in Classes I and II.

Let us mention, however, the existence of an infinite family of connected quartic half-arc-transitive metacirculants of Class IV, which are loosely attached, and thus not in Class I. They arise as certain $\mathbb{Z}_{p}$-covers, $p \geq 7$ a prime, in the following way. We start with $X=\mathcal{Z}(20,5 ; 9,2)$, a loosely attached half-arc-transitive metacirculant of Class IV, as our base graph. (Recall that the graphs $\mathcal{Z}(m, n ; r, t)$ were defined in Section 2.) Of the two possible Aut $X$-admissible orientations of the edges of $X$, we choose the one in which $u_{0}^{0}$ is the tail of the edges $u_{0}^{0} u_{1}^{0}$ and $u_{0}^{0} u_{9}^{0}$, and denote the corresponding oriented graph by $D_{X}$. Next, to every arc of $X$ assign voltage 1 or -1 in $\mathbb{Z}_{p}$ depending on whether its orientation is or is not compatible with the orientation of the corresponding edge in $D_{X}$. Call the obtained covering graph $\mathcal{C}_{p}(X)$. Using similar techniques as in Construction 5.10 one can show that $\mathcal{C}_{p}(X)$ is isomorphic to $\mathcal{Z}(20 p, 5 ; k, 2)$, where $k \in \mathbb{Z}_{20 p}$ is the unique element such that $k \equiv 9(\bmod 20)$ and $k \equiv 1(\bmod p)$. Moreover, one can also see that $\mathcal{C}_{p}(X)$ is a loosely attached half-arctransitive graph, thus giving an infinite family of half-arc-transitive metacirculants of Class IV which are not tightly attached. The technical details are omitted. 


\section{References}

1. Alspach, B., Parsons, T.D.: A construction for vertex-transitive graphs. Can. J. Math. 34, 307-318 (1982)

2. Alspach, B., Xu, M.-Y.: $\frac{1}{2}$-arc-transitive graphs of order 3p. J. Algebr. Comb. 3, 347-355 (1994)

3. Alspach, B., Marušič, D., Nowitz, L.: Constructing graphs which are $\frac{1}{2}$-transitive. J. Aust. Math. Soc. A 56, 391-402 (1994)

4. Biggs, N., White, A.T.: Permutation Groups and Combinatorial Structures. Cambridge University Press, Cambridge (1979)

5. Bondy, A., Murty, U.S.R.: Graph Theory with Applications. Elsevier, New York (1976)

6. Bouwer, I.Z.: Vertex and edge-transitive but not 1-transitive graphs. Can. Math. Bull. 13, 231-237 (1970)

7. Conder, M.D.E., Marušič, D.: A tetravalent half-arc-transitive graph with nonabelian vertex stabilizer. J. Comb. Theory B 88, 67-76 (2003)

8. D'Azevedo, A.B., Nedela, R.: Half-arc-transitive graphs and chiral hypermaps. Eur. J. Comb. 25, 423-436 (2004)

9. Dixon, J.D., Mortimer, B.: Permutation Groups. Springer, New York (1996)

10. Doyle, P.G.: On transitive graphs. Senior Thesis, Harvard College (1976)

11. Du, S.F., Xu, M.Y.: Vertex-primitive $\frac{1}{2}$-arc-transitive graphs of smallest order. Commun. Algebra 27, 163-171 (1998)

12. Feng, Y.Q., Kwak, J.H., Xu, M.Y., Zhou, J.X.: Tetravalent half-arc-transitive graphs of order $p^{4}$. Eur. J. Comb. (2007), doi:10.1016/j.ejc.2007.05.004

13. Feng, Y.Q., Wang, K.S., Zhou, C.X.: Constructing even radius tightly attached half-arc-transitive graphs of valency four. J. Algebr. Comb. 26, 431-451 (2007)

14. Feng, Y.Q., Wang, K.S., Zhou, C.X.: Tetravalent half-transitive graphs of order 4p. Eur. J. Comb. 28, 726-733 (2007)

15. Holt, D.F.: A graph which is edge transitive but not arc transitive. J. Graph Theory 5, 201-204 (1981)

16. Li, C.H., Sims, H.S.: On half-transitive metacirculant graphs of prime-power order. J. Comb. Theory B 81, 45-57 (2001)

17. Li, C.H., Lu, Z.P., Marušič, D.: On primitive permutation groups with small suborbits and their orbital graphs. J. Algebra 279, 749-770 (2004)

18. Malnič, A., Marušič, D.: Constructing 4-valent $\frac{1}{2}$-transitive graphs with a nonsolvable autmorphism group. J. Comb. Theory B 75, 46-55 (1999)

19. Malnič, A., Nedela, R., Škoviera, M.: Lifting graph automorphisms by voltage assignments. Eur. J. Comb. 21, 927-947 (2000)

20. Marušič, D.: Half-transitive group actions on finite graphs of valency 4. J. Comb. Theory B 73, 41-76 (1998)

21. Marušič, D.: Recent developments in half-transitive graphs. Discrete Math. 182, 219-231 (1998)

22. Marušič, D.: Quartic half-arc-transitive graphs with large vertex stabilizers. Discrete Math. 299, 180$193(2005)$

23. Marušič, D., Nedela, R.: Maps and half-transitive graphs of valency 4. Eur. J. Comb. 19, 345-354 (1998)

24. Marušič, D., Nedela, R.: On the point stabilizers of transitive permutation groups with non-self-paired suborbits of length 2. J. Group Theory 4, 19-43 (2001)

25. Marušič, D., Nedela, R.: Finite graphs of valency 4 and girth 4 admitting half-transitive group actions. J. Aust. Math. Soc. 73, 155-170 (2002)

26. Marušič, D., Pisanski, T.: Weakly flag-transitive configurations and $\frac{1}{2}$-transitive graphs. Eur. J. Comb. 20, 559-570 (1999)

27. Marušič, D., Praeger, C.E.: Tetravalent graphs admitting half-transitive group actions: alternating cycles. J. Comb. Theory B 75, 188-205 (1999)

28. Marušič, D., Waller, A.O.: Half-transitive graphs of valency 4 with prescribed attachment numbers. J. Graph Theory 34, 89-99 (2000)

29. Šparl, P.: A classification of tightly attached half-arc-transitive graphs of valency 4 (submitted)

30. Taylor, D.E., Xu, M.Y.: Vertex-primitive $\frac{1}{2}$-transitive graphs. J. Aust. Math. Soc. A 57, 113-124 (1994)

31. Thomassen, C., Watkins, M.E.: Infinite vertex-transitive, edge-transitive, non 1-transitive graphs. Proc. Am. Math. Soc. 105, 258-261 (1989) 
32. Tutte, W.T.: Connectivity in Graphs. University of Toronto Press, Toronto (1966)

33. Wang, R.J.: Half-transitive graphs of order a product of two distinct primes. Commun. Algebra 22, 915-927 (1994)

34. Wielandt, H.: Finite Permutation Groups. Academic, New York (1964)

35. Wilson, S.: Semi-transitive graphs. J. Graph Theory 45, 1-27 (2004)

36. Xu, M.Y.: Half-transitive graphs of prime cube order. J. Algebr. Comb. 1, 275-282 (1992)

37. Zhou, C.X., Feng, Y.Q.: An infinite family of tetravalent half-arc-transitive graphs. Discrete Math. 306, 2205-2211 (2006) 